\title{
XMM-Newton observations of the $\sigma$ Orionis cluster
}

\section{Spatial and spectral analysis of the full EPIC field $\star, \star \star$}

\author{
E. Franciosini ${ }^{1}$, R. Pallavicini ${ }^{1}$, and J. Sanz-Forcada ${ }^{2}$ \\ 1 INAF - Osservatorio Astronomico di Palermo, Piazza del Parlamento 1, 90134 Palermo, Italy \\ e-mail: francio@astropa.unipa.it \\ 2 Astrophysics Division - Research and Science Support Department of ESA, ESTEC, Postbus 299, 2200 AG Noordwijk, The Netherlands
}

Received 9 June 2005 / Accepted 15 September 2005

\section{ABSTRACT}

We present the results of an XMM-Newton observation of the young ( 2-4 Myr) cluster around the hot star $\sigma$ Orionis. In a previous paper we presented the analysis of the RGS spectrum of the central hot star; here we discuss the results of the analysis of the full EPIC field. We have detected $175 \mathrm{X}$-ray sources, 88 of which have been identified with cluster members, including very low-mass stars down to the substellar limit. We detected eleven new possible candidate members from the 2MASS catalogue. We find that late-type stars have a median $\log L_{\mathrm{X}} / L_{\mathrm{bol}} \sim-3.3$, i.e. very close to the saturation limit. We detected significant variability in $\sim 40 \%$ of late-type members or candidates, including 10 flaring sources; rotational modulation is detected in one K-type star and possibly in another 3 or 4 stars. Spectral analysis of the brightest sources shows typical quiescent temperatures in the range $T_{1} \sim 0.3-0.8 \mathrm{keV}$ and $T_{2} \sim 1-3 \mathrm{keV}$, with subsolar abundances $Z \sim 0.1-0.3 Z_{\odot}$, similar to what is found in other star-forming regions and associations. We find no significant difference in the spectral properties of classical and weak-lined T Tauri stars, although classical T Tauri stars tend to be less X-ray luminous than weak-lined T Tauri stars.

Key words. open clusters and associations: individual: $\sigma$ Orionis - stars: activity - stars: coronae - stars: pre-main sequence - stars: late-type - X-rays: stars

\section{Introduction}

The $\sigma$ Ori cluster was discovered by ROSAT (Wolk 1996; Walter et al. 1997) around the O9.5V binary star $\sigma$ Ori AB (which results from two components separated by $0.2^{\prime \prime}$ ). It belongs to the Orion OB1b association and is located at a distance of $352_{-85}^{+166}$ pc (from Hipparcos, ESA 1997). In addition to several hot stars, it is known to contain more than 100 likely pre-main sequence (PMS) late-type stars within 30' of $\sigma$ Ori (Wolk 1996; Sherry et al. 2004), as well as several brown dwarfs and planetary-mass objects (Béjar et al. 1999, 2001, 2004; Zapatero Osorio et al. 2000; Caballero et al. 2004). The estimated age of the cluster is 2-4 Myr (Zapatero Osorio et al. 2002; Oliveira et al. 2002; Sherry et al. 2004).

We have observed the $\sigma$ Ori cluster using XMM-Newton. The observation was centered on the hot star $\sigma$ Ori AB, in order to obtain both a high-resolution RGS spectrum of the central source and EPIC imaging data and low-resolution spectra over the whole field. The analysis of the RGS and EPIC

\footnotetext{
* Based on observations obtained with XMM-Newton, an ESA science mission with instruments and contributions directly funded by ESA Member States and NASA.

$\star \star$ Appendices are only available in electronic form at http://www.edpsciences.org
}

spectra of the hot star $\sigma$ Ori AB has been presented in a previous paper (Sanz-Forcada et al. 2004, hereafter referred to as Paper I), together with the analysis of the EPIC spectra of other three nearby bright sources that could potentially contaminate the RGS spectrum of $\sigma$ Ori AB. Of these, only the B2Vp star $\sigma$ Ori $\mathrm{E}$ was found to significantly affect the RGS spectrum: its EPIC spectrum allowed us to correct for its contribution, and therefore to derive the emission measure distribution and elemental abundances of $\sigma$ Ori AB. We found that $\sigma$ Ori AB has a much softer spectrum than the other sources, consistently with a wind origin, however the RGS spectrum shows no evidence for line broadenings and shifts with velocities $\gtrsim 800 \mathrm{~km} \mathrm{~s}^{-1}$, as could be produced by strong winds; the low $f / i$ line ratio in the $\mathrm{Ne}$ and $\mathrm{O} \mathrm{He}-$ like triplets indicates either high density (i.e. magnetic confinement either close to or far from the star) or, more likely, a strong UV radiation field (i.e. emission close to the star where the wind is however too weak to produce shocks).

In Paper I we also reported the detection of a strong flare from the magnetic hot star $\sigma$ Ori $\mathrm{E}$, which is not expected from models of X-ray emission from winds. Based on the characteristics of the flare and of the quiescent emission, whose spectrum is harder than that of $\sigma$ Ori $\mathrm{AB}$ and consistent with those 
of late-type stars, we argued that the flare and most of the quiescent emission is most likely due to an unseen late-type companion, although emission from the magnetic hot star itself cannot be excluded (see discussion in Paper I).

In this paper we present the analysis of the full EPIC field, in order to derive the X-ray properties of the other members or candidates of the $\sigma$ Ori cluster. The paper is organized as follows. X-ray observations and data analysis are described in Sect. 2. In Sect. 3 we discuss the X-ray properties of cluster members and compare our results with those of other star forming regions and young open clusters. Spectral analysis of the brightest sources is presented in Sect. 4. Discussion and conclusions are given in Sect. 5.

\section{Observations and data analysis}

XMM-Newton observations of the $\sigma$ Ori cluster, centered on the hot star $\sigma$ Ori AB, were carried out as part of the Guaranteed Time of one of us (R.P.) using both the EPIC MOS and PN cameras and the RGS instrument. The observation (ID 0101440301) started at 21:47 UT on March 23, 2002 and ended at 9:58 UT on March 24, 2002, for a total duration of 43 ks. The EPIC cameras were operated in Full Frame mode using the thick filter.

Data analysis was carried out using the standard tasks in SAS v.5.4.1. The analysis of the RGS data has been discussed in Paper I and will not be repeated here. EPIC calibrated and cleaned event files were derived from the raw data using the standard pipeline tasks EMCHAIN and EPCHAIN and then applying the appropriate filters to eliminate noise and bad events. The event files have also been time filtered in order to exclude a few short periods of high background due to proton flares; the final effective exposure time is $41 \mathrm{ks}$ for each MOS and $36 \mathrm{ks}$ for the PN. We limited our analysis to the $0.3-7.8 \mathrm{keV}$ energy band, since events below $0.3 \mathrm{keV}$ are mostly unrelated to bona-fide X-rays, while above $7.8 \mathrm{keV}$ only background is present. The combined EPIC (MOS1+MOS2+PN) image in the $0.3-7.8 \mathrm{keV}$ energy band is shown in Fig. 1. Exposure maps for each instrument in the same energy band were created using the task EEXPMAP.

Source detection was performed both on the individual datasets and on the merged MOS1+MOS2+PN dataset using the Wavelet Detection algorithm developed at INAFOsservatorio Astronomico di Palermo (Damiani et al. 1997; Damiani et al., in preparation), adapted to the EPIC case. From the comparison of the count rates of common sources detected on the individual datasets, we derived a median ratio of $\mathrm{PN}$ to MOS count rates of $\sim 3.2$; this value was then used as a scaling factor for the PN exposure map in the detection on the summed dataset, in order to take into account the different sensitivities of the PN and MOS cameras. This implies a resulting MOS equivalent exposure time of $\sim 200 \mathrm{ks}$ for the merged dataset. Count rates derived from the detection on the summed dataset are MOS equivalent count rates.

We used a significance detection threshold of $5 \sigma$, chosen in order to have at most one spurious detection, and determined from a set of 100 simulations of pure background datasets with the same number of counts as the observation. After removing

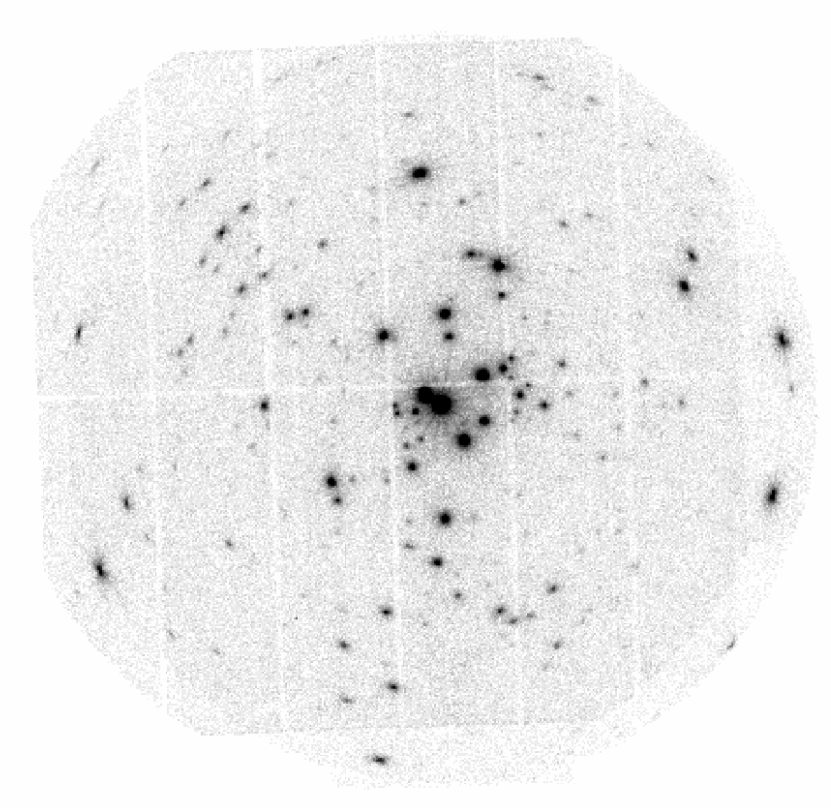

Fig. 1. Composite EPIC (MOS1+MOS2+PN) image of the $\sigma$ Ori cluster.

a few obviously spurious detections (due to hot pixels, to the point spread function structure of the central bright source, and to out-of-time events) we obtained a total of 175 sources, three of which were detected only on a single instrument dataset (two on MOS and one on PN).

\subsection{The optical catalogue}

The $\sigma$ Ori cluster has been the subject of several studies after its discovery by ROSAT (Walter et al. 1997). Wolk (1996) performed follow-up UBVRI photometry down to $V \sim 18$ within $30^{\prime}$ from $\sigma$ Ori, finding $\sim 130$ candidate PMS stars, and obtained medium-resolution spectroscopy for a few of them. Recently, Sherry et al. (2004) extended the survey to an area of $\sim 0.89 \mathrm{deg}^{2}$ around the hot star down to $V \sim 20$. Several deep optical and infrared surveys of the cluster, with limiting magnitude $I \sim 22-24$, have led to the discovery of several very-low mass stars and brown dwarfs as well as of a number of planetary-mass objects (Béjar et al. 1999, 2001, 2004; Zapatero Osorio et al. 2000; Caballero et al. 2004). Additional photometric RI surveys down to $I \sim 18-19$ have been performed by Scholz \& Eislöffel (2004) and Kenyon et al. (2005). Low- and medium-resolution spectroscopic observations are available for a subsample of low-mass stars and brown dwarfs (Béjar et al. 1999; Barrado y Navascués et al. 2001, 2003; Martín et al. 2001; Zapatero Osorio et al. 2002; Kenyon et al. 2005; Burningham et al. 2005).

We constructed our optical catalogue by including all stars from the above-mentioned studies, plus a few additional $\mathrm{H} \alpha$ emission line stars from Weaver \& Babcock (2004) and the hot stars from the studies of the Orion OB association by Warren \& Hesser (1977) and Brown et al. (1994). After crosscorrelating the data from the available studies, we obtain a total of 266 stars falling in the XMM field of view. About $40 \%$ of 
the late-type stars in the catalogue have spectroscopic membership information based on radial velocity, lithium or other youthness indicators. For the remaining stars only photometry or at most $\mathrm{H} \alpha$ measurements are avalaible: in these cases, we have assigned a photometric membership based on their position in different colour-magnitude diagrams, rejecting as members those stars falling below the $10 \mathrm{Myr}$ isochrone in at least two diagrams. For stars without a spectroscopically-determined spectral type, we derived spectral types from their $R-I, V-I$ or $I-J$ colours using the transformations by Kenyon \& Hartmann (1995) and Leggett et al. (2001). In total, there are 218 probable or candidate members in our catalogue, including 8 earlytype (O-B-A) stars, two late F-G type stars, $143 \mathrm{~K}$ - and early M-type stars $(\lesssim \mathrm{M} 4)$, and 65 very-low mass stars and brown dwarfs with spectral type later than $\sim \mathrm{M} 5^{1}$.

Most of the stars in our optical catalogue have counterparts in the 2MASS All-Sky Point Source Catalogue (Cutri et al. 2003): for these stars we used their 2MASS coordinates in order to have accurate positions.

\subsection{Source identification}

In order to find optical counterparts to our X-ray source list, we have determined the optimal search radius by constructing the cumulative distribution of the offsets between X-ray and optical position, following Randich \& Schmitt (1995). We chose a search radius of $5^{\prime \prime}$, for which less than two spurious identifications are expected. After correlating the X-ray source list with the optical catalogue, we found a median offset of $\sim 1.4^{\prime \prime}$ in right ascension between the X-ray and optical positions; we therefore corrected the X-ray positions, and repeated the identification process. We found 88 sources with at least one cluster member or candidate within 5" (including two double identifications); these sources are listed in Table A.1 together with their optical properties. The position of detected members in the $V-(V-I)$ and $I-(R-I)$ colour-magnitude diagrams are shown in Fig. 2. X-ray detections delineate very clearly the cluster sequence, confirming the importance of X-ray emission as a membership indicator for young stars.

Of the detected members, three are $\mathrm{OB}$ stars, i.e. $\sigma$ Ori $\mathrm{AB}$ (O9.5V), $\sigma$ Ori E (B2Vp) and HD 294272 (B9.5III), and one is an A8V star (HD 37564). We have detected the two FG-type stars, $\sim 64 \%$ of K-type stars and $\sim 50 \%$ of early-M stars $(<\mathrm{M} 5)$. Seven sources $(\sim 11 \%)$ have been identified with very lowmass stars and brown dwarfs of spectral type later than $\sim \mathrm{M} 5$. However, in two cases, the identification is ambiguous, since there is another early-M star falling inside the identification radius. One of these cases is source NX 67, which is identified with SE $70(\sim \mathrm{M} 4)$ at $2.6^{\prime \prime}$, and S Ori 68, a planetary-mass object of spectral type L5.0 (Béjar et al. 2001), at a distance of $4.7^{\prime \prime}$. This source underwent a flare during the observation, with an increase in the count rate by a factor of $\sim 4$ and a total duration of $\sim 10 \mathrm{ks}$ (see Sect. 3.2, Fig. 7). Inspection of the $\mathrm{X}$-ray image (Fig. 3) shows that the bulk of the X-ray emission,

${ }^{1}$ Note that, at the age of $\sigma$ Ori, the substellar limit lies around spectral type $\sim$ M 5-M 6, depending on the adopted model isochrones (see e.g. Zapatero Osorio et al. 2002).
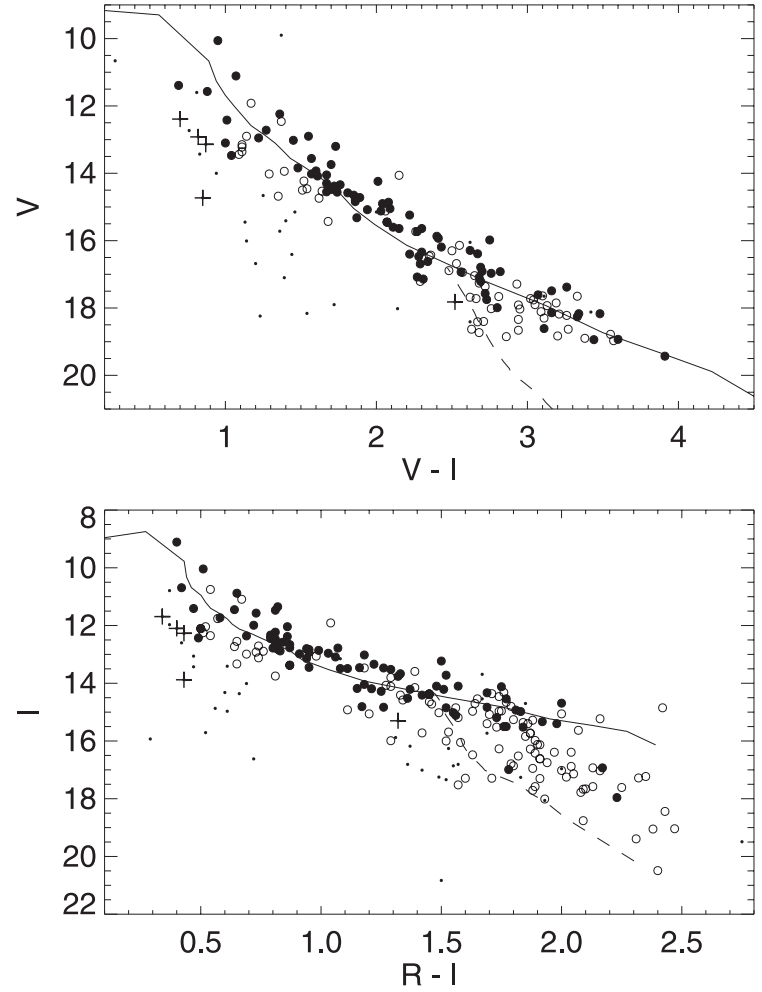

Fig. 2. $V$ vs. $(V-I)$ and $I$ vs. $(R-I)$ colour-magnitude diagrams for stars in the XMM-Newton field of view. Detected cluster members and candidates are marked with filled circles, while open circles indicate non detections. Crosses and dots mark the position of detected and undetected non-members, respectively. The solid and dashed lines are the $5 \mathrm{Myr}$ isochrones from Siess et al. (2000) and Baraffe et al. (1998), respectively, shifted for the distance and reddening of the $\sigma$ Ori cluster.

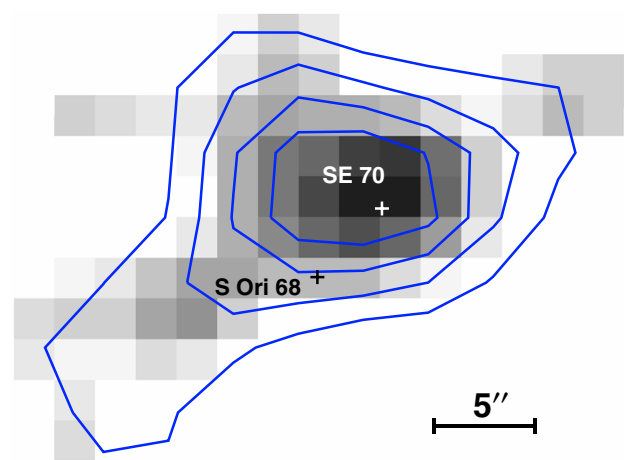

Fig. 3. Close-up of the EPIC summed image at the position of the X-ray source identified with S Ori 68 and SE 70. The positions of the two stars are marked with crosses. Contour levels are at 0.2, 0.3, 0.4, $0.5 \mathrm{cts} \operatorname{arcsec}^{-2}$.

and therefore the flare, is associated with SE 70, although we cannot exclude the presence of a very weak contribution from S Ori 68 itself. Unfortunately the statistics outside of the flare is too low to allow us to derive any information on the relative contribution of the two objects to the quiescent X-ray source. In the analysis of Sect. 3.1 we have therefore assigned all the X-ray flux to SE 70, while taking for S Ori 68 the same value as upper limit to its emission. 
Table 1. X-ray sources identified with possible cluster candidates from 2MASS. NX is a running identification number for the X-ray sources. The column labeled "Sign." indicates the significance of detection.

\begin{tabular}{rcrrccccc}
\hline \hline NX & RA $_{X} \begin{array}{c}\text { Dec }_{X} \\
(J 2000)\end{array}$ & Sign. & $\begin{array}{r}\text { Count rate } \\
(\mathrm{cts} / \mathrm{ks})\end{array}$ & 2MASS & $\begin{array}{c}\Delta r \\
\left({ }^{\prime}\right)\end{array}$ & $J$ & $J-K$ \\
\hline 14 & $5: 38: 15.63$ & $-2: 42: 05.5$ & 12.9 & $2.67 \pm 0.39$ & J05381552-0242051 & 0.36 & 16.33 & \\
42 & $5: 38: 31.07$ & $-2: 34: 02.8$ & 7.0 & $0.64 \pm 0.13$ & J05383098-0234038 & 1.00 & 14.92 & 0.91 \\
50 & $5: 38: 33.05$ & $-2: 39: 27.2$ & 8.2 & $0.53 \pm 0.11$ & J05383302-0239279 & 1.30 & 14.59 & 0.89 \\
91 & $5: 38: 48.47$ & $-2: 36: 42.3$ & 6.6 & $0.74 \pm 0.14$ & J05384828-0236409 & 1.86 & 12.04 & 0.90 \\
101 & $5: 38: 50.97$ & $-2: 27: 44.4$ & 6.4 & $0.70 \pm 0.17$ & J05385101-0227456 & 2.35 & 14.28 & 0.95 \\
103 & $5: 38: 51.80$ & $-2: 36: 02.6$ & 28.2 & $3.50 \pm 0.25$ & J05385173-0236033 & 0.84 & 12.91 & 0.88 \\
116 & $5: 38: 59.13$ & $-2: 34: 15.5$ & 7.7 & $0.63 \pm 0.13$ & J05385884-0234131 & 3.71 & 15.86 & 0.97 \\
148 & $5: 39: 13.58$ & $-2: 37: 37.8$ & 10.4 & $1.18 \pm 0.18$ & J05391346-0237391 & 1.31 & 13.41 & 0.91 \\
151 & $5: 39: 15.89$ & $-2: 36: 49.7$ & 7.4 & $0.74 \pm 0.15$ & J05391582-0236507 & 1.15 & 13.25 & 1.03 \\
153 & $5: 39: 17.12$ & $-2: 41: 17.6$ & 22.7 & $4.20 \pm 0.37$ & J05391699-0241171 & 0.59 & 14.29 & 0.92 \\
175 & $5: 39: 40.24$ & $-2: 43: 07.9$ & 7.5 & $1.94 \pm 0.46$ & J05393998-0243097 & 3.04 & 10.65 & 1.12 \\
\hline
\end{tabular}

The other source with two possible counterparts is NX 167, which has been identified with S Ori J053926.8-022614, a $\sim$ M 6 star, at 3.9", and SE $94(\sim \mathrm{M} 2)$ at 3.2". In this case, it is not possible to identify which star is the most probable X-ray emitting one; we have therefore equally divided the X-ray flux between them.

The faintest star with a certain X-ray detection is the candidate brown dwarf S Ori 25, which has a spectral type M7.5 (Barrado y Navascués et al. 2003) and an estimated mass of $\sim 0.04 M_{\odot}$ (Muzerolle et al. 2003). Its X-ray luminosity in the $0.3-8 \mathrm{keV}$ band is $L_{\mathrm{X}} \sim 3 \times 10^{28} \mathrm{erg} \mathrm{s}^{-1}$ (see Sect. 3.1), corresponding to $\log L_{\mathrm{X}} / L_{\mathrm{bol}} \sim-3.3$, i.e. close to the saturation limit.

For the remaining 124 late-type ( $\mathrm{F}$ to $\mathrm{M}$ ) cluster members and candidates with no associated X-ray source we determined $3 \sigma$ upper limits at the optical positions using the Wavelet algorithm. Their X-ray and optical properties are given in Table A.2.

Six additional X-ray sources have been identified with probable cluster non members from our optical catalogue, including an early-type star (HD 37525, B5V) which was rejected as member of the OB association by Warren \& Hesser (1977) on the basis of proper motion. Interestingly, this source underwent a flare at the end of the observation, after $\sim 10 \mathrm{hrs}$ of quiescent emission; an analysis of its quiescent PN spectrum shows high temperatures $(0.6$ and $1.3 \mathrm{keV})$, similar to those found for the $\mathrm{B} 2 \mathrm{Vp}$ star $\sigma$ Ori $\mathrm{E}$ (Paper I) and for late-type stars (see Sect. 4), suggesting that the emission might originate from an unseen late-type companion. Of the other non members, one (SWW 222) has a radial velocity inconsistent with cluster membership (Burningham et al. 2005), while the others have been rejected on the basis of photometry only. These $\mathrm{X}$-ray sources are listed in Table 2.

In order to increase the number of identifications, we have also cross-correlated the X-ray source list with the 2MASS and the GSC2. 2 catalogues, finding 13 counterparts from 2MASS and 2 from GSC2. Eleven of the 2MASS counterparts have $J H K$ photometry consistent with cluster membership, and are therefore considered as possible candidate members of the cluster; we list them in Table 1. The other identified X-ray sources are given in Table 2 .

For the remaining 66 sources (listed in Table B.1) we did not find any known counterpart in any astronomical catalogue. This number is consistent with the expected number of extragalactic X-ray sources $(\sim 60-80)$ in the direction of $\sigma$ Ori, derived using the $\log N-\log S$ relations by Hasinger et al. (2001) and Tozzi et al. (2001).

\section{Results}

\subsection{X-ray luminosities}

In order to derive X-ray luminosities, we have computed a conversion factor in the $0.3-8 \mathrm{keV}$ band using the results of the PN and MOS1 spectral fits of late-type stars (see Sect. 4). The conversion factor has been determined by comparing the count rates obtained from the Wavelet algorithm on the summed dataset with the unabsorbed X-ray flux derived from the bestfit models, excluding the few sources with absorption higher than the mean value $N_{\mathrm{H}}=2.7 \times 10^{20} \mathrm{~cm}^{-2}$, derived from the mean reddening $E(B-V)=0.05$ (Lee 1968; Brown et al. 1994), and then taking the median value. The derived conversion factor, valid for the summed and MOS datasets, is $\mathrm{CF}=6.6 \times 10^{-12} \mathrm{erg} \mathrm{cm}^{-2} \mathrm{cnt}^{-1}$, with an uncertainty of $\sim 15 \%$ ( $1 \sigma$ standard deviation). For the source detected only in the $\mathrm{PN}$ we derived in the same way a median conversion factor $\mathrm{CF}=2.1 \times 10^{-12} \mathrm{erg} \mathrm{cm}^{-2} \mathrm{cnt}^{-1}$ for the PN count rates. We applied these factors to all stars for which no spectral analysis can be performed, assuming that all of them have the mean absorption, since individual absorption measures are generally not available. For stars having $N_{\mathrm{H}} \sim 1-2 \times 10^{21} \mathrm{~cm}^{-2}$, as found for some sources, this will underestimate the X-ray luminosity by a factor of $\sim 1.5$. For the stars with spectral fits (Table 3 and Paper I) we used instead the fluxes determined from the PN or MOS1 (if PN is not available) best-fit models. Finally, X-ray luminosities have been derived using the Hipparcos cluster distance of $352 \mathrm{pc}$.

The sensitivity in the center of the field (from $3 \sigma$ upper limits) is $L_{\mathrm{X}} \sim 2 \times 10^{28} \mathrm{erg} \mathrm{s}^{-1}$, and decreases to 
Table 2. X-ray sources identified with probable cluster non-members or with stars without membership information. NX is a running identification number for the X-ray sources. The column labeled "Sign." indicates the significance of detection. Optical identifications labeled 4771-... and r05... are from Wolk (1996); SWW 222 is from Sherry et al. (2004).

\begin{tabular}{rcrrllllll}
\hline \hline NX & RAX $_{(\mathrm{J} 2000)}$ & Dec $_{\mathrm{X}}$ & Sign. & $\begin{array}{r}\text { Count rate } \\
(\mathrm{cts} / \mathrm{ks})\end{array}$ & Optical ID & $\begin{array}{c}\Delta r \\
\left({ }^{\prime \prime}\right)\end{array}$ & $I$ & $R-I$ & Notes \\
\hline 41 & $5: 38: 30.06$ & $-2: 23: 36.6$ & 26.1 & $7.50 \pm 0.59$ & r053829-0223 & 1.66 & 12.27 & 0.43 & $a$ \\
46 & $5: 38: 32.21$ & $-2: 32: 43.6$ & 13.7 & $1.14 \pm 0.15$ & GSC2 S02003215575 & 0.62 & & & \\
47 & $5: 38: 32.70$ & $-2: 31: 16.1$ & 6.4 & $0.57 \pm 0.13$ & 2MASS J05383268-0231156 & 1.29 & & & \\
56 & $5: 38: 34.30$ & $-2: 34: 59.9$ & 21.9 & $3.52 \pm 0.35$ & r053834-0234 & 1.63 & 13.88 & 0.43 & $a$ \\
114 & $5: 38: 57.20$ & $-2: 31: 25.5$ & 7.2 & $0.65 \pm 0.13$ & $4771-1071$ & 1.74 & 12.10 & 0.40 & $a$ \\
119 & $5: 38: 59.61$ & $-2: 45: 08.6$ & 38.3 & $9.36 \pm 0.53$ & 4771-0026 & 0.68 & 11.69 & 0.34 & $a$ \\
120 & $5: 38: 59.65$ & $-2: 35: 27.9$ & 9.1 & $0.76 \pm 0.16$ & 2MASS J05385930-0235282 & 3.83 & & & \\
123 & $5: 39: 01.19$ & $-2: 33: 37.8$ & 13.8 & $1.42 \pm 0.18$ & GSC2 S02003215312 & 4.70 & & & \\
125 & $5: 39: 01.58$ & $-2: 38: 57.0$ & 132.8 & $45.72 \pm 0.91$ & HD 37525 & 0.67 & 8.07 & & $b$ \\
169 & $5: 39: 30.72$ & $-2: 38: 28.7$ & 10.1 & $1.77 \pm 0.29$ & SWW 222 & 1.93 & 15.30 & 1.32 & $c$ \\
\hline
\end{tabular}

${ }^{a}$ Probable non member on the basis of photometry.

${ }^{b}$ Non member from proper motion (Warren \& Hesser 1977).

${ }^{c}$ Non member from radial velocity (Burningham et al. 2005).

$\sim 4 \times 10^{28} \mathrm{erg} \mathrm{s}^{-1}$ at $13^{\prime}$ offaxis, and to $\sim 7 \times 10^{28} \mathrm{erg} \mathrm{s}^{-1}$ where only MOS is present.

Figure 4 shows $L_{\mathrm{X}}$ and $L_{\mathrm{X}} / L_{\mathrm{bol}}$ as a function of the $R-I$ colour for cluster members and candidates. For early-type stars, we find $\log L_{\mathrm{X}} / L_{\mathrm{bol}} \sim-6.6$ for the 09.5 star $\sigma$ Ori AB, consistent with the typical value $(\sim-7)$ found for hot stars (Pallavicini et al. 1981; Berghöfer et al. 1997), while $\log L_{\mathrm{X}} / L_{\mathrm{bol}} \sim-5.5$ for the other early-type stars and for the F7 star. For late-type stars there is a general trend of decreasing $L_{X}$ with increasing colour, although there is a scatter of more than one order of magnitude at each colour. Correspondingly, $L_{\mathrm{X}} / L_{\mathrm{bol}}$ is nearly constant, as observed in other young clusters and starforming regions (SFRs; e.g. Feigelson et al. 2002; Flaccomio et al. 2003b,d). The median value is $\log L_{\mathrm{X}} / L_{\mathrm{bol}} \sim-3.3$ for detected stars, and $\log L_{\mathrm{X}} / L_{\mathrm{bol}} \sim-3.4$ taking into account also upper limits, i.e. very close to the saturation limit. This value is consistent with the results obtained for other SFRs of similar age (e.g. Flaccomio et al. 2003d). There are a few stars lying significantly above the saturation value $\log L_{\mathrm{X}} / L_{\mathrm{bol}} \sim-3$ : this is generally due to the presence of flares or to strong variability during the observation.

In Fig. 5 we show the X-ray luminosity distribution function (XLDF) for K and early-M stars (upper panel). The XLDF confirms the decrease of X-ray luminosity with spectral type: $\mathrm{K}$-type stars are clearly more luminous than M-type stars, with a median $\log L_{\mathrm{X}}=29.73 \mathrm{erg} \mathrm{s}^{-1}$ for $\mathrm{K}$ stars, an order of magnitude higher than the median $\log L_{\mathrm{X}}=28.77 \mathrm{erg} \mathrm{s}^{-1}$ for M-type stars.

For the subsample of stars with available $\mathrm{H} \alpha$ measurements we also compared the XLDFs for classical (CTTS) and weak-lined (WTTS) T Tauri stars. We have classified stars as CTTS or WTTS using the $\mathrm{H} \alpha$ EW criterium derived by Barrado y Navascués \& Martín (2003), which depends on spectral type. In the bottom panels of Fig. 5 we plot the XLDFs for CTTS and WTTS separately for stars earlier than M 5, and for stars and brown dwarfs of all spectral types. In the first case, the two distributions are clearly different, with a median

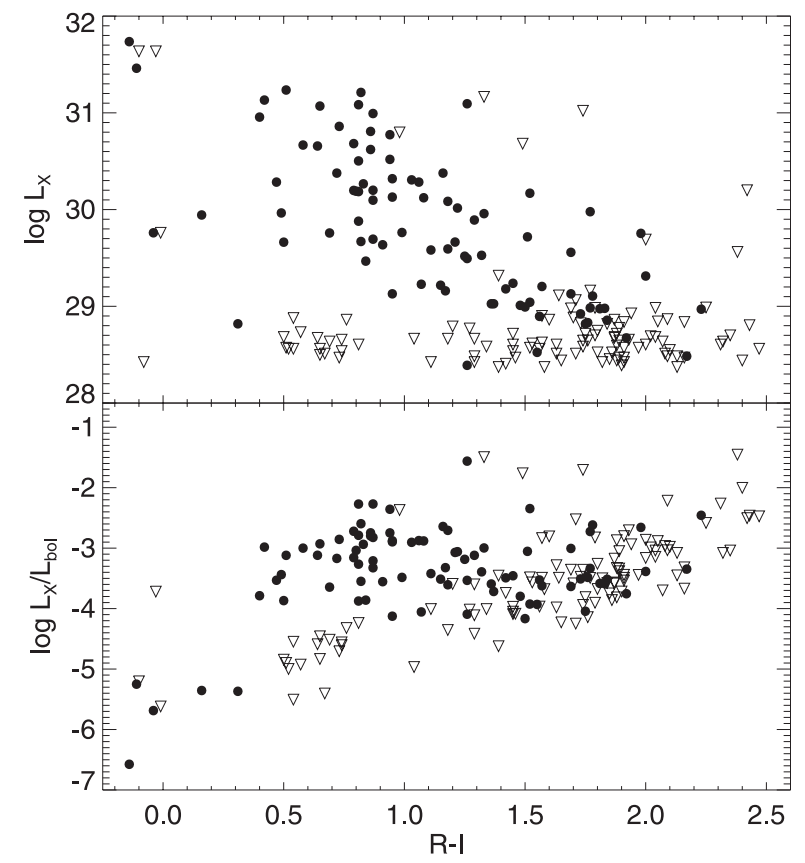

Fig. 4. $L_{\mathrm{X}}$ (top) and $L_{\mathrm{X}} / L_{\mathrm{bol}}$ (bottom) as a function of the $(R-I)$ colour for detected (dots) and undetected (open triangles) cluster members and candidates.

$\log L_{\mathrm{X}}=30.12 \mathrm{erg} \mathrm{s}^{-1}$ for WTTS and $\log L_{\mathrm{X}}=29.47 \mathrm{erg} \mathrm{s}^{-1}$ for CTTS. As a check, we have performed a series of twosample tests, as implemented in ASURV Rev. 1.1 (Lavalley et al. 1992), finding a probability $P=0.04-0.11$ that the two distributions are drawn from the same population. If one considers all stars and brown dwarfs, the two distributions do not appear to be significantly different, although CTTS are still a factor of $\sim 2$ less luminous (median $\log L_{X} \sim 28.76$ ) than WTTS (median $\log L_{\mathrm{X}} \sim 29.11$ ). In this latter case the two-sample tests are inconclusive, giving $P=0.7-0.9$. The different results found in the two cases can be attributed to the higher number of upper limits in the full sample of stars and brown dwarfs: in fact, 

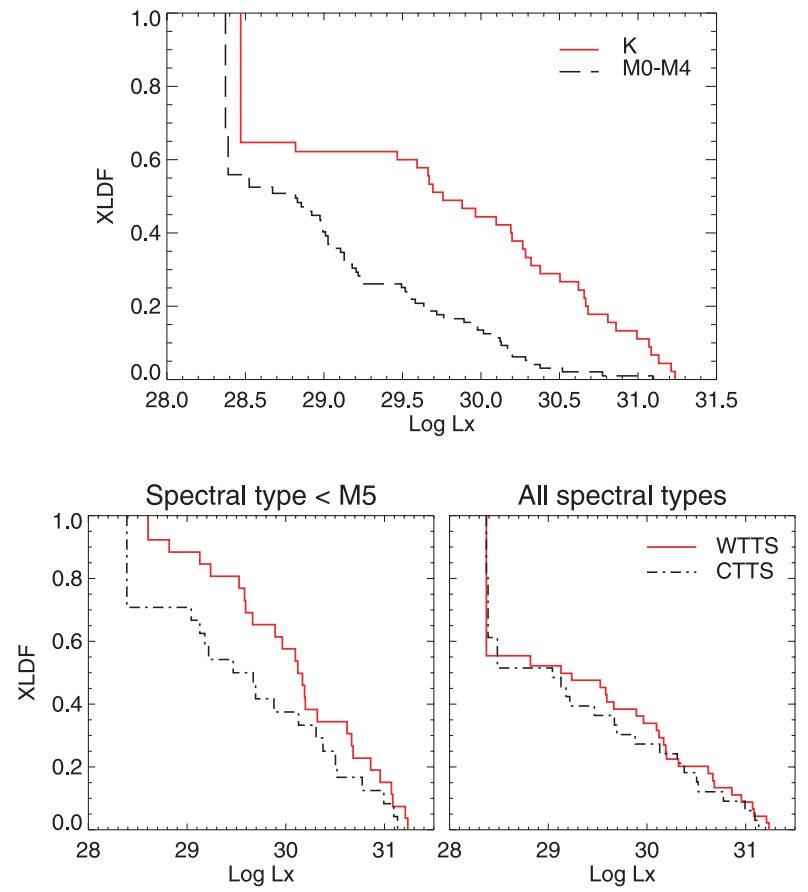

Fig. 5. Upper panel: comparison of the XLDF for K-type (red solid line) and early M-type (black dashed line) stars in $\sigma$ Ori. Lower panels: comparison of the XLDFs for WTTS (red solid line) and CTTS (black dot-dashed line), considering only stars earlier than $\sim \mathrm{M} 5$ (left) and all stars and candidate brown dwarfs (right).

for spectral types later than $\sim$ M 5 we detected only one CTTS out of 9 and none of the 19 WTTS, compared with a detection rate of 18/24 ( 75\%) and 24/27 ( 90\%) for CTTS and WTTS earlier than M 5, respectively.

In Fig. 6 we compare the XLDFs of K and M-type stars of $\sigma$ Ori with those of other SFRs and associations of similar age. We have used for the comparison the data of $\rho$ Oph (1 Myr), Orion Nebula Cluster (ONC, 1-2 Myr) and Chameleon I (4 Myr) reanalyzed by Flaccomio et al. (2003d, see their paper for details). Since the luminosities derived by these authors are in the $0.1-4 \mathrm{keV}$ band, we have computed an appropriate conversion factor using the same procedure described above, in order to derive luminosities in the same band. We find that, for K-type stars, the high-luminosity tail $\left(\log L_{X}>30.5\right)$ is intermediate between that of ONC and those of $\rho$ Oph and Cha I, and the median luminosity appears to be significantly lower than ONC and Cha I (by a factor of $\sim 3-5$ ), but comparable to that of $\rho$ Oph. The two sample tests indicate that the XLDF is significantly different from that of ONC $(P<0.002)$ but are inconclusive in the other cases. On the other hand, for M-type stars the high-luminosity tails of all the SFRs are comparable, but the median for $\sigma$ Ori $\left(\log L_{\mathrm{X}}=28.9\right)$ is significantly lower than those of all the other SFRs $\left(\log L_{X}=29.4-29.7\right)$; the twosample tests confirm that the XLDFs are not drawn by the same population, giving in all cases $P<0.0002$.

\subsection{Variability}

Light curves for all detected members or candidates have been extracted from the photon event lists using circular regions of

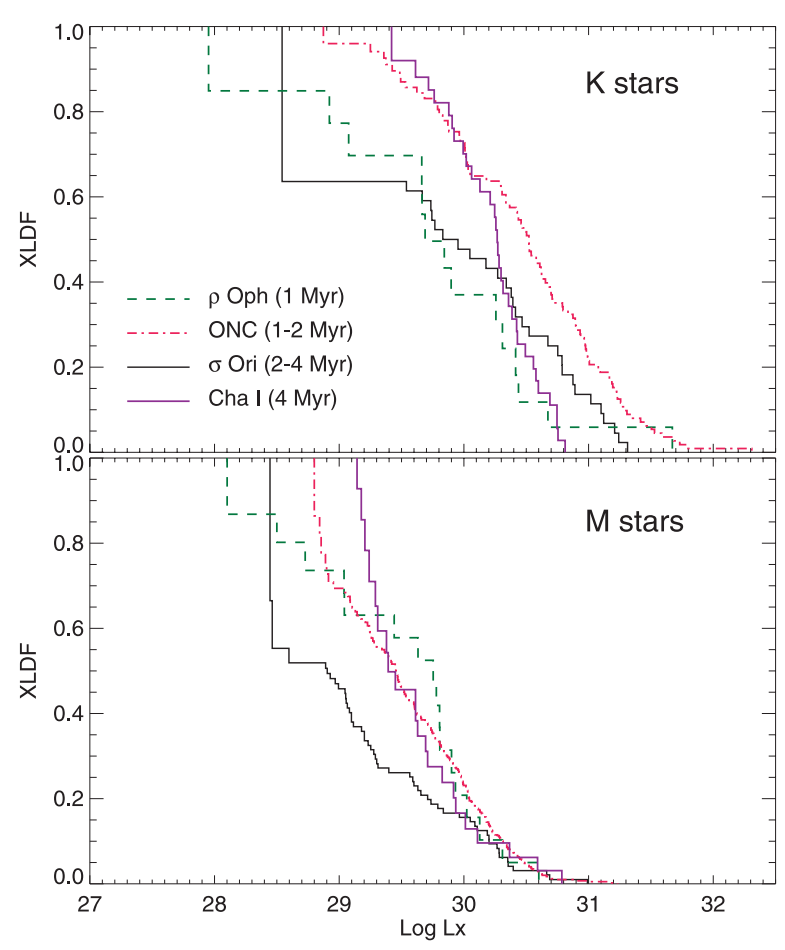

Fig. 6. Comparison of the XLDF of $\sigma$ Ori with those of other SFRs and associations of similar age for $\mathrm{K}$ and early $\mathrm{M}$ stars.

radius $24^{\prime \prime}$ centered on the source position, except for a few cases where we used smaller radii to avoid overlap with nearby close sources. Inspection of the light curves reveals that many of the detected members or candidates show significant variability during our observation, including the strong X-ray flare detected on the B2Vp star $\sigma$ Ori E and discussed in Paper I. In order to quantify the variability of the sources, we have performed a Kolmogorov-Smirnov test on the unbinned photon arrival times, using data from PN, or from MOS for sources falling on PN CCD gaps or outside of the PN field of view. We find that $\sim 35 \%$ (29/84) of late-type members are variable at the $99 \%$ confidence level, and other 7 stars (8\%) are possibly variable at the $95 \%$ level. In particular, $47 \%$ of the CTTS and $54 \%$ of the WTTS are variable at the $99 \%$ level. In addition, variability at the $99 \%$ level is detected in two of the 2MASS candidates of Table 1. Ten late-type sources show clear flares, including one of the 2MASS candidates, with increases in the count rates by factors of $\sim 4-20$ in $\sim 1-2 \mathrm{~h}$ and decay times of $\sim 2-10 \mathrm{~h}$. The combined PN+MOS1+MOS2 light curves of these flaring sources (espressed as MOS equivalent count rates) are shown in Fig. 7. The other variable sources show generally low-level flare-like variability or slow variations. In Fig. 8 we show some examples of light curves of sources with strong variability not clearly attributable to flares. One source (NX 76) shows a steady decay by a factor of $\sim 2$ during the entire observation, that could be attributed either to the decay of a long-lasting flare or to rotational modulation. Sources NX 62 and NX 64 have very high temperatures (see Table 3), but their light curve does not show the typical behaviour of flares: in both cases a steady emission level for a few hours is observed after a slow rise or before a slow decay. A similar behaviour 

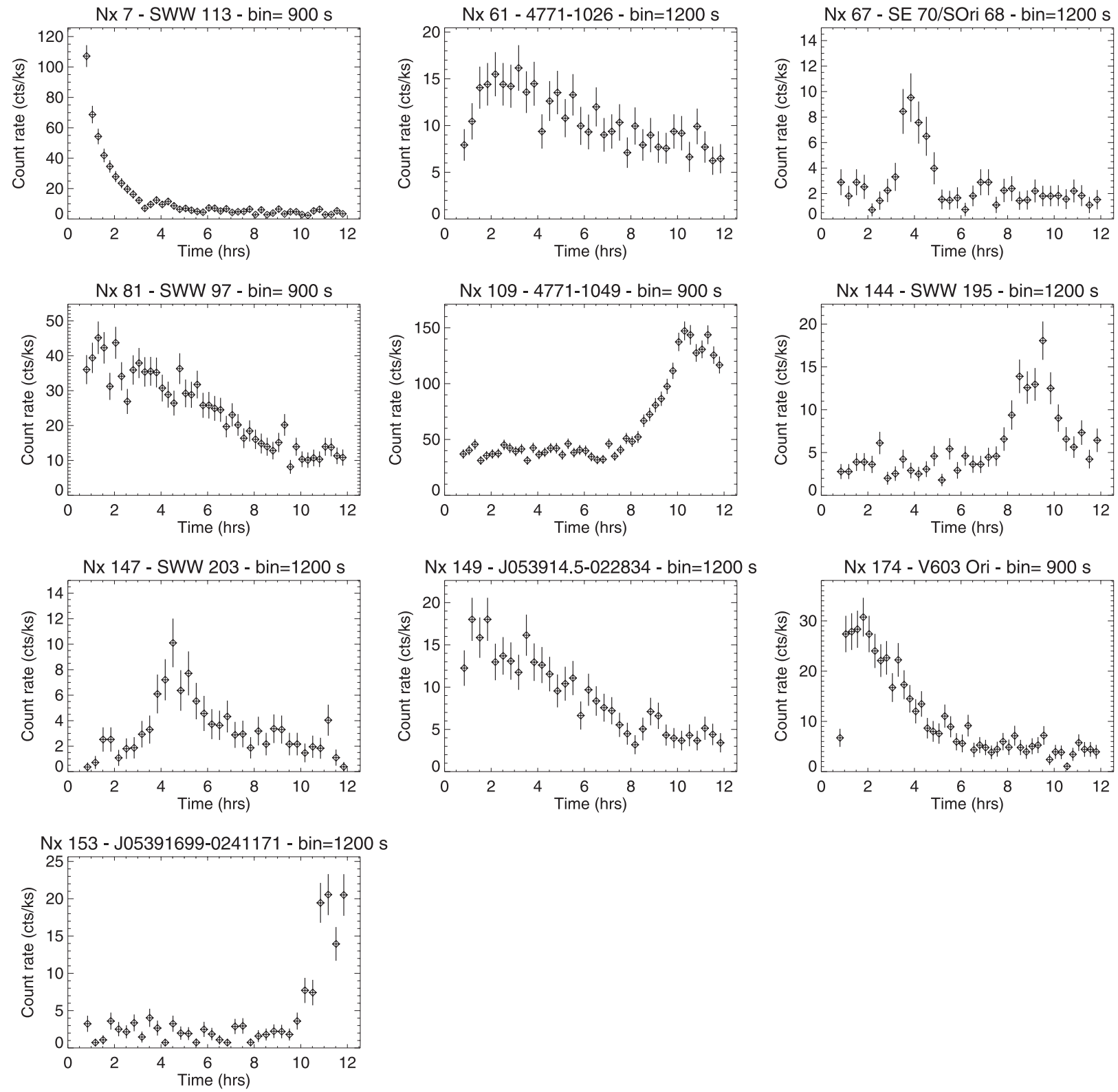

Fig. 7. Combined PN+MOS1+MOS2 light curves of cluster members or candidates showing strong flares during our observation, including a candidate member from 2MASS (last panel). Count rates are espressed as MOS equivalent count rates. The different bin size used for each source is indicated at the top of each panel together with the source identification.

is observed for NX 79. All these light curves could be interpreted as due to rotational modulation of the emission from active, possibly flaring regions unevenly distributed on the stellar surface (Stelzer et al. 1999). A more evident modulation effect is instead present in source NX 70 (source \#4 in Paper I), which shows two peaks followed by a similar decay trend. A fit of the light curve with a simple sinusoidal model gives a period of $\sim 9.2 \pm 0.1 \mathrm{~h}$, with an amplitude variation of $\sim 25 \%$ around the mean. The folded light curve is shown in the bottom right panel of Fig. 8, where we have marked with different symbols data points belonging to different periods: there is a perfect overlap between the light curves in the two periods. We caution, however, that, having data available only for slightly more than one period, these results are not conclusive, and we cannot completely exclude that the observed variability is due to the occurrence of two similar flaring events rather than to rotational modulation.

\section{Spectral analysis}

For the detected cluster members or candidates having at least 500 cts in the PN or MOS1 we performed spectral analysis. We excluded sources falling on CCD gaps, that have an effective low number of counts despite their derived high count rate from the wavelet detection. A detailed PN and MOS spectral analysis of the four bright central sources has already been performed in Paper I; however, we have reanalyzed the spectra of the two central K-type stars (NX65 = source \#3 of Paper I, and NX70 = source \#4) in order to compare in a consistent 
Table 3. Results of the 2-T or 1-T spectral fitting of the PN and/or MOS1 spectra of sources with more than 500 counts. Errors are $90 \%$ confidence ranges for one interesting parameter.

\begin{tabular}{|c|c|c|c|c|c|c|c|c|c|c|c|}
\hline NX & \multicolumn{2}{|c|}{$(\mathrm{keV})$} & \multicolumn{2}{|c|}{$\left(10^{53} \mathrm{~cm}^{-3}\right)$} & $Z / Z_{\odot}$ & $N_{\mathrm{H}}^{a}$ & $\chi_{\mathrm{r}}^{2} /$ d.o.f. & $F_{\mathrm{X}}^{b}$ & Inst. $^{c}$ & $\begin{array}{l}\text { CTT/ } \\
\text { WTT }\end{array}$ & Notes \\
\hline 2 & $0.75_{-0.13}^{+0.09}$ & $1.63_{-0.26}^{+0.44}$ & $5.19_{-2.55}^{+5.84}$ & $8.90_{-3.20}^{+4.09}$ & $0.25_{-0.10}^{+0.17}$ & & $0.56 / 59$ & 9.10 & M & CTT? & \\
\hline 3 & $0.83_{-0.06}^{+0.15}$ & $2.39_{-0.61}^{+1.03}$ & $12.83_{-2.88}^{+2.71}$ & $6.64_{-1.47}^{+1.73}$ & $0.16_{-0.05}^{+0.07}$ & & $0.62 / 74$ & 11.55 & M & WTT? & \\
\hline 7 & $4.83_{-1.42}^{+2.86}$ & & $3.75_{-0.76}^{+1.01}$ & & $0.56_{-0.49}^{+0.72}$ & $5.79_{-1.32}^{+1.89}$ & $0.67 / 34$ & 3.99 & $\mathrm{P}$ & CTT & flare \\
\hline \multirow[t]{2}{*}{8} & $0.61_{-0.22}^{+0.14}$ & $1.25_{-0.16}^{+0.19}$ & $1.69_{-0.56}^{+1.10}$ & $3.40_{-1.30}^{+1.47}$ & $0.23_{-0.08}^{+0.13}$ & & $0.63 / 80$ & 3.05 & $\mathrm{P}$ & & \\
\hline & $0.43_{-0.14}^{+0.36}$ & $1.02_{-0.50}^{+0.31}$ & $2.11_{-0.98}^{+1.45}$ & $3.65_{-1.35}^{+1.68}$ & $0.14_{-0.06}^{+0.06}$ & & $0.97 / 24$ & 2.64 & M & & \\
\hline 34 & $0.80_{-0.14}^{+0.20}$ & & $1.88_{-0.40}^{+0.50}$ & & $0.02_{-0.02}^{+0.04}$ & & $0.51 / 21$ & 0.61 & $\mathrm{P}$ & & \\
\hline 39 & $0.56_{-0.25}^{+0.22}$ & $1.26_{-0.25}^{+0.37}$ & $0.56_{-0.25}^{+0.40}$ & $1.14_{-0.30}^{+0.40}$ & $0.12_{-0.06}^{+0.13}$ & & $0.59 / 39$ & 0.82 & $\mathrm{P}$ & & \\
\hline 49 & $0.29_{-0.05}^{+0.07}$ & $1.01_{-0.10}^{+0.19}$ & $1.11_{-0.44}^{+0.64}$ & $1.54_{-0.47}^{+0.58}$ & $0.19_{-0.07}^{+0.13}$ & & $0.64 / 60$ & 1.24 & $\mathrm{P}$ & & \\
\hline 61 & $1.92_{-0.49}^{+0.72}$ & & $2.28_{-0.93}^{+1.71}$ & & $0.29_{-0.26}^{+0.59}$ & $5.00_{-1.52}^{+2.76}$ & $0.47 / 19$ & 1.60 & $\mathrm{P}$ & CTT & IR exc.,flare \\
\hline 62 & $1.06_{-0.11}^{+0.17}$ & $7.69_{-2.63}^{+6.43}$ & $9.76_{-0.87}^{+2.00}$ & $3.90_{-0.55}^{+0.62}$ & $0.05_{-0.02}^{+0.03}$ & & $0.50 / 80$ & 8.14 & M & WTT & \\
\hline \multirow[t]{2}{*}{64} & $0.80_{-0.05}^{+0.06}$ & $6.59_{-2.48}^{+8.26}$ & $4.18_{-0.55}^{+0.56}$ & $1.13_{-0.19}^{+0.24}$ & $0.08_{-0.03}^{+0.04}$ & & $0.57 / 131$ & 2.82 & $\mathrm{P}$ & WTT & no IR exc. \\
\hline & $0.80_{-0.13}^{+0.19}$ & $6.59^{d}$ & $3.91_{-1.14}^{+1.16}$ & $1.04_{-0.43}^{+0.33}$ & $0.07_{-0.04}^{+0.05}$ & & $0.51 / 40$ & 2.53 & M & & \\
\hline \multirow[t]{2}{*}{65} & $0.94_{-0.04}^{+0.04}$ & $3.65_{-0.55}^{+0.67}$ & $8.84_{-0.68}^{+0.68}$ & $4.34_{-0.49}^{+0.43}$ & $0.12_{-0.03}^{+0.03}$ & & $0.69 / 298$ & 7.89 & $\mathrm{P}$ & WTT & \\
\hline & $0.97_{-0.24}^{+0.11}$ & $3.63_{-0.86}^{+1.58}$ & $13.97_{-9.09}^{+4.12}$ & $4.48_{-0.80}^{+8.17}$ & $0.06_{-0.02}^{+0.03}$ & & $0.74 / 111$ & 9.49 & M & & \\
\hline 69 & $0.30_{-0.05}^{+0.05}$ & $1.22_{-0.28}^{+0.15}$ & $3.88_{-1.10}^{+0.90}$ & $2.61_{-0.65}^{+3.04}$ & $0.11_{-0.07}^{+0.07}$ & $1.88_{-0.37}^{+0.32}$ & $0.62 / 43$ & 2.22 & $\mathrm{P}$ & CTT & IR exc. \\
\hline \multirow[t]{2}{*}{70} & $0.76_{-0.04}^{+0.04}$ & $2.54_{-0.20}^{+0.26}$ & $1.93_{-0.46}^{+0.70}$ & $6.32_{-0.42}^{+0.43}$ & $0.35_{-0.09}^{+0.11}$ & & $0.81 / 283$ & 6.60 & $\mathrm{P}$ & CTT & \\
\hline & $0.80_{-0.08}^{+0.24}$ & $3.01_{-0.54}^{+0.71}$ & $4.08_{-2.14}^{+4.24}$ & $6.36_{-0.73}^{+0.85}$ & $0.17_{-0.10}^{+0.20}$ & & $0.68 / 95$ & 7.25 & M & & \\
\hline \multirow[t]{2}{*}{76} & $0.78_{-0.09}^{+0.07}$ & $2.46_{-0.89}^{+1.75}$ & $1.19_{-0.61}^{+0.99}$ & $0.79_{-0.21}^{+0.28}$ & $0.21_{-0.10}^{+0.22}$ & & $0.70 / 70$ & 1.29 & $P$ & & \\
\hline & $0.71_{-0.13}^{+0.13}$ & $1.37_{-0.41}^{+0.70}$ & $1.10_{-0.59}^{+0.86}$ & $1.13_{-0.42}^{+0.87}$ & $0.20_{-0.11}^{+0.20}$ & & $0.42 / 20$ & 1.29 & M & & \\
\hline \multirow[t]{2}{*}{78} & $0.33_{-0.06}^{+0.08}$ & $1.29_{-0.10}^{+0.06}$ & $1.94_{-0.52}^{+0.55}$ & $4.91_{-0.65}^{+0.79}$ & $0.15_{-0.05}^{+0.06}$ & & $0.67 / 133$ & 3.23 & $\mathrm{P}$ & WTT & \\
\hline & $0.33_{-0.11}^{+0.51}$ & $1.33_{-0.15}^{+0.29}$ & $1.32_{-0.67}^{+0.87}$ & $4.67_{-1.41}^{+1.44}$ & $0.17_{-0.09}^{+0.23}$ & & $0.89 / 40$ & 3.05 & M & & \\
\hline \multirow[t]{2}{*}{79} & $0.79_{-0.07}^{+0.05}$ & $1.94_{-0.28}^{+0.46}$ & $2.61_{-0.80}^{+1.42}$ & $4.26_{-0.86}^{+0.59}$ & $0.20_{-0.06}^{+0.08}$ & & $0.72 / 177$ & 4.32 & $\mathrm{P}$ & & \\
\hline & $0.77_{-0.21}^{+0.29}$ & $1.84_{-0.49}^{+0.82}$ & $3.51_{-1.45}^{+1.40}$ & $4.33_{-0.85}^{+1.10}$ & $0.09_{-0.06}^{+0.10}$ & & $0.88 / 52$ & 4.05 & M & & \\
\hline 81 & $0.86_{-0.11}^{+0.15}$ & $3.21_{-0.61}^{+0.89}$ & $0.18_{-0.09}^{+0.25}$ & $1.28_{-0.30}^{+0.27}$ & $0.87_{-0.47}^{+0.86}$ & & $0.62 / 57$ & 1.60 & $\mathrm{P}$ & & flare \\
\hline \multirow[t]{2}{*}{90} & $0.97_{-0.13}^{+0.07}$ & $3.31_{-0.77}^{+0.64}$ & $8.17_{-3.60}^{+4.82}$ & $8.20_{-3.66}^{+1.68}$ & $0.14_{-0.08}^{+0.19}$ & $1.64_{-0.33}^{+0.45}$ & $0.56 / 181$ & 10.91 & $\mathrm{P}$ & WTT & IR exc. \\
\hline & $0.93_{-0.20}^{+0.15}$ & $3.35_{-0.77}^{+1.10}$ & $9.15_{-5.34}^{+8.82}$ & $9.45_{-1.48}^{+1.93}$ & $0.12_{-0.08}^{+0.33}$ & $1.91_{-0.55}^{+0.74}$ & $0.73 / 66$ & 12.07 & M & & \\
\hline 92 & $0.32_{-0.04}^{+0.07}$ & $1.49_{-0.31}^{+0.23}$ & $0.52_{-0.25}^{+0.43}$ & $0.93_{-0.36}^{+0.52}$ & $0.47_{-0.30}^{+0.65}$ & & $0.70 / 54$ & 1.07 & $\mathrm{P}$ & & \\
\hline \multirow[t]{2}{*}{93} & $0.63_{-0.13}^{+0.14}$ & $1.27_{-0.18}^{+0.25}$ & $0.76_{-0.27}^{+0.53}$ & $1.17_{-0.61}^{+0.74}$ & $0.32_{-0.11}^{+0.12}$ & & $0.51 / 74$ & 1.36 & $\mathrm{P}$ & CTT & no IR exc. \\
\hline & $0.85_{-0.08}^{+0.15}$ & & $3.37_{-1.22}^{+1.11}$ & & $0.08_{-0.03}^{+0.05}$ & & $0.45 / 22$ & 1.41 & M & & \\
\hline 104 & $0.37_{-0.16}^{+0.32}$ & $0.84_{-0.12}^{+0.21}$ & $1.16_{-0.62}^{+0.80}$ & $1.78_{-0.76}^{+0.71}$ & $0.09_{-0.05}^{+0.11}$ & & $0.37 / 28$ & 1.04 & $\mathrm{P}$ & WTT & \\
\hline 106 & $0.61_{-0.26}^{+0.13}$ & $1.43_{-0.32}^{+0.55}$ & $0.47_{-0.24}^{+0.39}$ & $0.74_{-0.24}^{+0.39}$ & $0.35_{-0.18}^{+0.41}$ & & $0.56 / 31$ & 0.89 & $\mathrm{P}$ & WTT & \\
\hline 109 & $0.98_{-0.15}^{+0.13}$ & $6.84_{-2.78}^{+10.68}$ & $5.84_{-1.08}^{+1.17}$ & $2.08_{-0.33}^{+0.37}$ & $0.10_{-0.04}^{+0.20}$ & & $0.74 / 58$ & 4.86 & M & WTT & flare \\
\hline 110 & $0.93_{-0.13}^{+0.12}$ & & $7.07_{-2.37}^{+2.85}$ & & $0.09_{-0.05}^{+0.07}$ & & $0.68 / 20$ & 3.12 & M & WTT & \\
\hline 122 & $0.65_{-0.24}^{+0.21}$ & $1.27_{-0.26}^{+0.55}$ & $0.24_{-0.13}^{+0.43}$ & $0.58_{-0.25}^{+0.43}$ & $0.38_{-0.21}^{+0.51}$ & & $0.44 / 26$ & 0.62 & $P$ & WTT? & \\
\hline 132 & $0.73_{-0.32}^{+0.22}$ & $1.67_{-0.56}^{+1.55}$ & $0.58_{-0.36}^{+1.25}$ & $0.74_{-0.27}^{+0.47}$ & $0.24_{-0.17}^{+0.34}$ & & $0.40 / 30$ & 0.84 & $\mathrm{P}$ & WTT & no IR exc. \\
\hline 138 & $0.84_{-0.16}^{+0.27}$ & $2.86_{-0.69}^{+4.92}$ & $0.77_{-0.52}^{+2.52}$ & $2.02_{-1.16}^{+0.55}$ & $0.26_{-0.22}^{+0.64}$ & $2.08_{-0.72}^{+1.43}$ & $0.68 / 42$ & 2.14 & $\mathrm{P}$ & CTT? & \\
\hline 145 & $0.23_{-0.04}^{+0.09}$ & $1.06_{-0.13}^{+0.18}$ & $2.19_{-1.26}^{+2.18}$ & $1.36_{-0.19}^{+0.46}$ & $0.19_{-0.10}^{+0.43}$ & $1.32_{-0.99}^{+2.21}$ & $0.44 / 36$ & 1.40 & $\mathrm{P}$ & WTT & no IR exc. \\
\hline 149 & $1.21_{-0.40}^{+0.72}$ & & $2.08_{-0.85}^{+2.08}$ & & $0.08_{-0.07}^{+0.12}$ & $1.48_{-0.72}^{+1.11}$ & $0.51 / 19$ & 0.99 & $\mathrm{P}$ & WTT & IR exc.?,flare \\
\hline 150 & $0.85_{-0.09}^{+0.16}$ & & $1.29_{-0.33}^{+0.34}$ & & $0.11_{-0.05}^{+0.08}$ & & $0.69 / 22$ & 0.59 & $\mathrm{P}$ & & A8V \\
\hline 156 & $0.56_{-0.16}^{+0.14}$ & $1.23_{-0.22}^{+0.85}$ & $0.80_{-0.25}^{+0.55}$ & $1.14_{-0.42}^{+0.44}$ & $0.30_{-0.13}^{+0.23}$ & & $0.81 / 38$ & 1.29 & $\mathrm{P}$ & & \\
\hline 170 & $0.36_{-0.09}^{+0.37}$ & $1.24_{-0.27}^{+0.32}$ & $0.64_{-0.33}^{+0.49}$ & $0.99_{-0.44}^{+0.70}$ & $0.33_{-0.21}^{+0.69}$ & & $0.34 / 24$ & 1.03 & $\mathrm{P}$ & & \\
\hline 172 & $0.74_{-0.08}^{+0.05}$ & $2.02_{-0.30}^{+0.54}$ & $2.03_{-0.65}^{+0.93}$ & $4.73_{-0.76}^{+0.76}$ & $0.54_{-0.18}^{+0.33}$ & & $0.85 / 105$ & 6.07 & $\mathrm{P}$ & WTT & G5, no IR exc. \\
\hline 174 & $5.44_{-1.54}^{+5.58}$ & & $7.47_{-1.45}^{+1.59}$ & & $0.74_{-0.64}^{+0.85}$ & $28.5_{-8.0}^{+9.0}$ & $0.54 / 24$ & 8.38 & $\mathrm{P}$ & CTT & IR exc.,flare \\
\hline
\end{tabular}

${ }^{a}$ Hydrogen column density in units of $10^{21} \mathrm{~cm}^{-2}$. Where no value is given, it was kept fixed at $N_{\mathrm{H}}=2.7 \times 10^{20} \mathrm{~cm}^{-2}$.

${ }^{b}$ Unabsorbed X-ray flux in the $0.3-8 \mathrm{keV}$ band, in units of $10^{-13} \mathrm{erg} \mathrm{cm}^{-2} \mathrm{~s}^{-1}$.

${ }^{c}$ Instrument from which the spectrum was extracted (P for PN, M for MOS1).

${ }^{d} T_{2}$ was kept fixed to the PN fit value since it was not constrained by the MOS1 fit. 

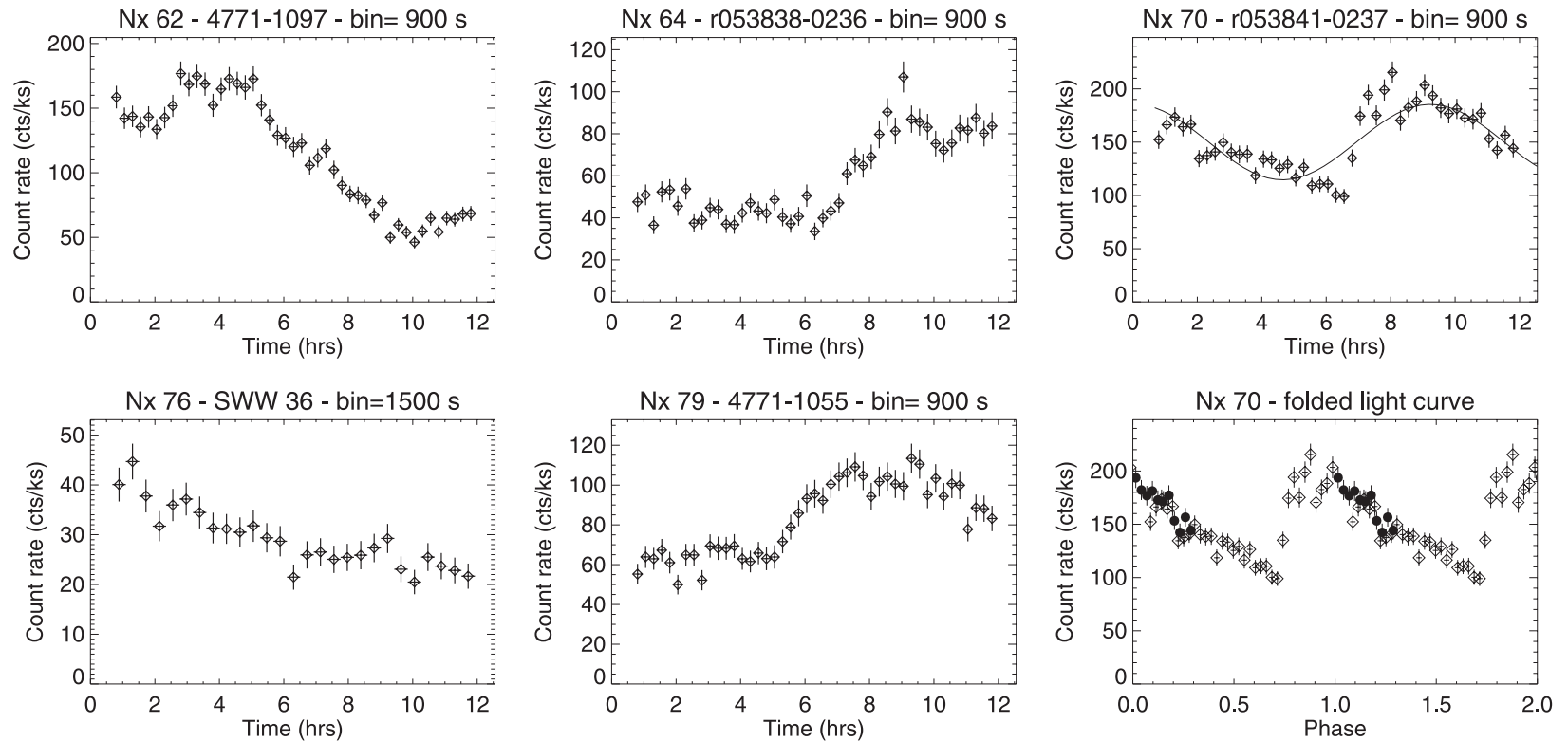

Fig. 8. Combined PN+MOS1+MOS2 light curves of cluster members or candidates showing significant variability not clearly attributable to flares. Note in particular the modulation of source NX 70 (right panels). The top right panel shows the light curve of NX 70 together with a best-fit sinusoidal curve (solid line). In the bottom right panel we show the same light curve folded with the derived rotational period of $9.2 \mathrm{~h}$, where different symbols mark data from the first (diamonds) and second (filled circles) periods; for clarity two rotational phases are shown.

way their properties with those of the other late-type sources discussed in this paper.

PN and/or MOS1 spectra have been extracted from the same circular regions used for the light curves. Background spectra for each source were extracted from a nearby circular region free from other X-ray sources and on the same CCD chip, using the same extraction radius as the corresponding source region. Response matrices and ancillary files were generated for each source using the standard SAS tasks RMFGEN and ARFGEN. Spectra have been rebinned to have at least 20 counts per bin, and were fitted in XSPEC v.11.3.0 in the energy range $0.3-8 \mathrm{keV}$, using a two-temperature APEC v.1.3.0 model with variable global abundance. For most of the weakest sources with $<1000$ cts, acceptable fits were found with only one temperature component. The hydrogen column density was generally kept fixed at the value $N_{\mathrm{H}}=2.7 \times 10^{20} \mathrm{~cm}^{-2}$, derived from the measured reddening $E(B-V)=0.05$. In a few cases however this led to an unacceptable fit at low energies, and therefore we left the column density as a free parameter, obtaining best-fit values of $N_{\mathrm{H}} \sim 1.3-2 \times 10^{21} \mathrm{~cm}^{-2}$ for quiescent sources, i.e. higher by about one order of magnitude, and even higher values $\left(N_{\mathrm{H}} \geq 5 \times 10^{21} \mathrm{~cm}^{-2}\right)$ for three flaring sources. We note that the eight sources needing a higher column density are either CTTS or stars with known IR excess; the only exception is source NX 145, identified with the star 4771-1038, a WTTS with no IR excess (Oliveira et al. 2004).

The resulting best-fit parameters are given in Table 3 , and a sample of the spectra together with the best-fit models are shown in Fig. 9 for quiescent sources (with high and low absorption) and in Fig. 10 for the three flaring CTT stars with higher absorption. We find typical quiescent temperatures of $T_{1} \sim 0.3-0.8 \mathrm{keV}$ and $T_{2} \sim 1-3 \mathrm{keV}$, in agreement with the results found for other young clusters and SFRs (e.g. Feigelson \& Montmerle 1999). In two cases (NX 62 and NX 64) temperatures up to $6-8 \mathrm{keV}$ are found, although it is not clear from their light curves whether these sources are flaring during the observation (see Fig. 8). Emission measures range between $10^{52}$ and $10^{54} \mathrm{~cm}^{-3}$, with $E M_{2} / E M_{1} \sim 0.5-2.5$. In all cases we find subsolar abundances, with most stars having $Z \sim 0.1-0.3 Z_{\odot}$, as commonly observed in active stars. Somewhat higher abundances $\left(Z \sim 0.6-0.9 Z_{\odot}\right)$ are found for three flaring sources. For the small subsample of WTTS and CTTS we find no significant difference in the coronal parameters of the two classes: in particular we do not find the higher spread in CTTS abundances with respect to WTTS found in L1551 by Favata et al. (2003).

Particularly interesting is source NX 174, identified with V603 Ori, a Class II star with strong IR excess and source of the protostellar jet HH 445 (Oliveira \& van Loon 2004). This source underwent a strong flare during the observation: the spectrum is quite hot, with $T \sim 7 \mathrm{keV}$, showing an evident Fe $6.7 \mathrm{keV}$ line, and the emission is strongly suppressed below $1.5 \mathrm{keV}$, implying a column density $N_{\mathrm{H}}=2.8 \times 10^{22} \mathrm{~cm}^{-2}$, i.e. two orders of magnitude higher than the mean cluster absorption.

\section{Discussion and conclusions}

In this paper we have presented the spatial analysis of an XMM-Newton observation of the $\sigma$ Ori cluster centered on the hot star $\sigma$ Ori AB. We have detected half of the early-type (O-A) stars, including $\sigma$ Ori AB, $65 \%$ of F-K stars and $\sim 50 \%$ of early M (i.e. M 0-M 4) stars above a detection limit of $\sim 2 \times$ $10^{28} \mathrm{erg} \mathrm{s}^{-1}$. Our detection rates for late-type stars are lower than what observed in other SFRs and very young clusters, 

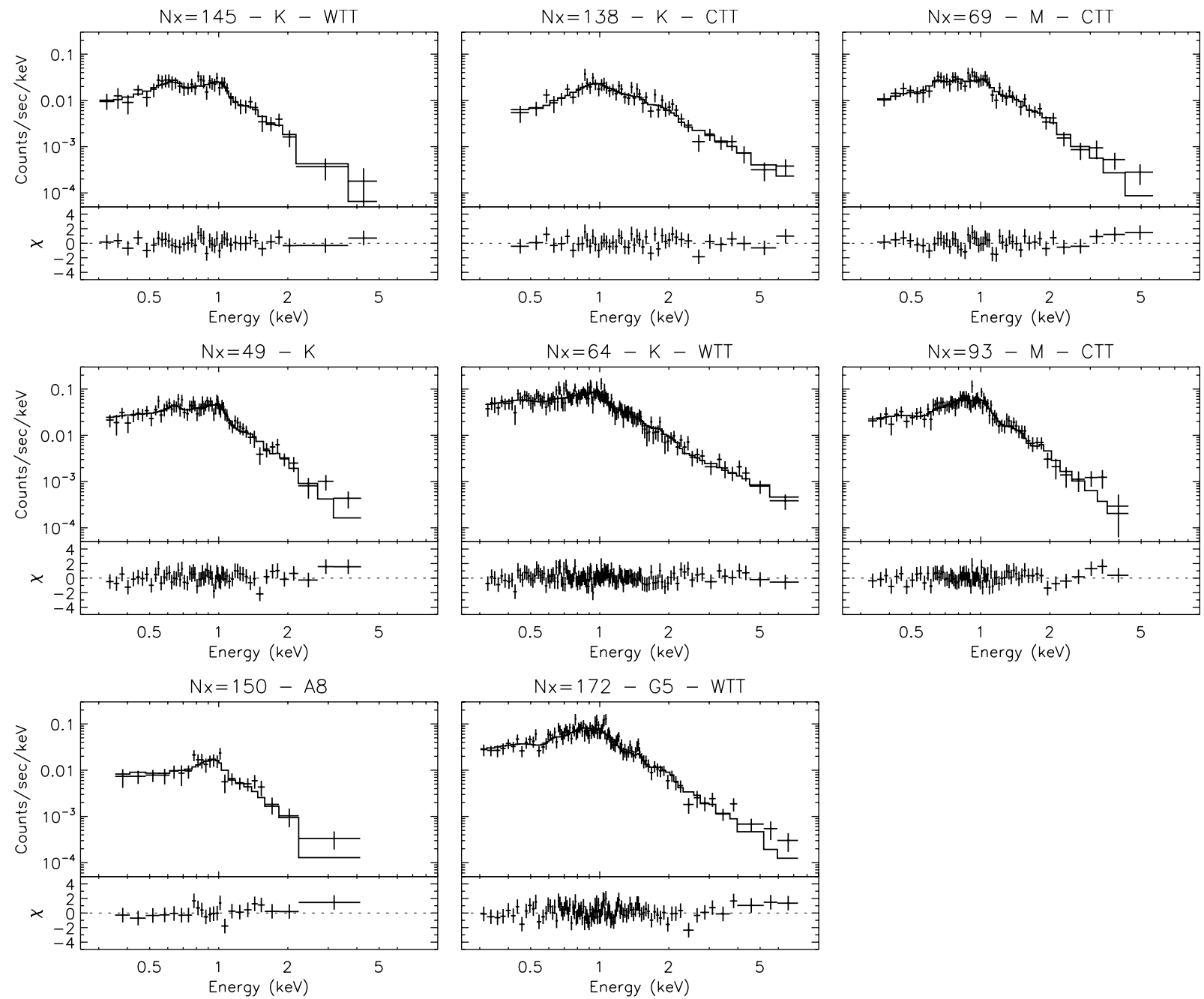

Fig. 9. Top and middle panels: examples of PN spectra of K-type and M-type cluster members with high $\left(N_{\mathrm{H}} \sim 1-2 \times 10^{21} \mathrm{~cm}^{-2}\right.$, top panels) and low $\left(N_{\mathrm{H}}=2.7 \times 10^{20} \mathrm{~cm}^{-2}\right.$, middle panels $)$ absorption. For each $N_{\mathrm{H}}$ case, two spectra are shown for K-type stars, one corresponding to the coolest $(\langle T\rangle \sim 0.6 \mathrm{keV}$, left $)$ and the other to the hottest $(\langle T\rangle \sim 2 \mathrm{keV}$, center) coronal temperatures; for M-type stars we show in both cases typical spectra with $\langle T\rangle \sim 1 \mathrm{keV}$ (right). Bottom panels: PN spectra of the A8 and the G5 stars. Note the similarity of both spectra with those of later type stars.
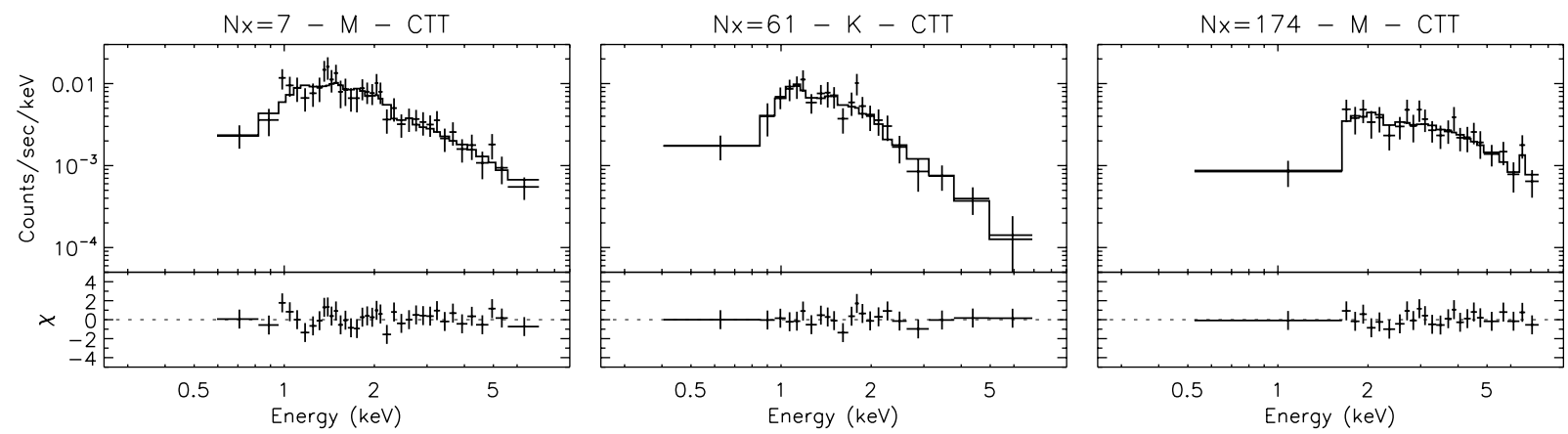

Fig. 10. PN spectra of the three CTT stars showing strong flares with high absorption $\left(N_{\mathrm{H}} \geq 5 \times 10^{21} \mathrm{~cm}^{-2}\right)$. Note in particular, for source NX 174, the very high absorption that strongly suppresses all emission below $1.5 \mathrm{keV}$, and the evident Fe $6.7 \mathrm{keV}$ line.

where $\sim 80 \%$ of late-type stars are generally detected above similar levels (e.g. Garmire et al. 2000; Preibisch \& Zinnecker 2001; Flaccomio et al. 2003a,d). A possible explanation for this discrepancy might be contamination of our optical sample by non-members. While other regions have well established membership lists, in the case of $\sigma$ Ori only $\sim 50 \%$ of the candidate and probable members have been confirmed spectroscopically. On the other hand, it is well known that PMS stars are powerful X-ray emitters, with luminosities $10-10^{4}$ times higher than older late-type stars (Feigelson \& Montmerle 1999), and the presence of strong X-ray emission is generally used to identify new members of SFRs and young clusters. It is therefore likely 
that most of the undetected candidates might turn out to be older field stars rather than young cluster members. In particular, there are 45 undetected cluster candidates that have been selected on the basis of photometry only: if most, or all, of them were indeed non-members, we would raise the detection rate of late-type stars to $\sim 75-80 \%$, bringing our results in agreement with other observations. Optical spectroscopic observations are needed (and in fact have been recently obtained by us using the multi-object spectrograph FLAMES at the VLT; data analysis is in progress) in order to determine the membership status of these stars and to check whether the lower detection rate found in $\sigma$ Ori is due to the inclusion of non-members in the sample, or it reflects a true difference from other SFRs.

We have found that for late-type stars $L_{X}$ declines towards later spectral types, while $L_{\mathrm{X}} / L_{\mathrm{bol}}$ is nearly constant, as commonly observed in other young clusters and SFRs. The spread of 1.5-2 orders of magnitude observed at all colours in both $L_{\mathrm{X}}$ and $L_{\mathrm{X}} / L_{\mathrm{bol}}$ is consistent with earlier results (e.g. Feigelson $\&$ Montmerle 1999; Preibisch \& Zinnecker 2002). The median $\log L_{\mathrm{X}} / L_{\mathrm{bol}}$ is $\sim-3.3$, i.e. very close to the saturation limit. This value is higher than the value of $\sim-4$ generally found for young ( $\$ 2$ Myr) SFRs (Preibisch \& Zinnecker 2001; Feigelson et al. 2002, 2003; Skinner et al. 2003; Flaccomio et al. 2003d), but is consistent with the observed increase of $L_{\mathrm{X}} / L_{\mathrm{bol}}$ with age for stars with $M<M_{\odot}$, reaching the saturation value of $10^{-3}$ at 4 Myr (Flaccomio et al. 2003d; Stelzer et al. 2004).

The comparison of the XLDFs of $\sigma$ Ori late-type stars with those of other SFRs of similar age shows that the median X-ray luminosity of $\sigma$ Ori late-type stars is significantly lower than the other SFRs. Our results appear to be in disagreement with Flaccomio et al. (2003d), who found no evidence for a significant evolution of the median X-ray luminosity of pre-main sequence stars with $M=0.25-2 M_{\odot}$ up to $\sim 10 \mathrm{Myr}$. However, this disagreement could be significantly reduced, or removed, if we consider the higher percentage of upper limits in our sample and the likely contamination by non-members, as discussed above. For example, if we exclude all undetected candidate members with only photometry available, the XLDF of $\sigma$ Ori becomes similar to the other ones, in agreement with the lack of PMS evolution found by Flaccomio et al. (2003d).

About $40 \%$ of the detected members show significant variability, with $\sim 10 \%$ undergoing strong flares during our observation. Strong variability is commonly observed in up to $\sim 50 \%$ of PMS stars in SFRs and very young clusters. The frequency of flares is comparable to what found e.g. in IC 348 (Preibisch \& Zinnecker 2002), NGC 1333 (Getman et al. 2002) and NGC 2264 (Ramírez et al. 2004), but it is much lower than in $\rho$ Oph, where $\sim 35 \%$ of T Tauri stars were found to be flaring by XMM-Newton in an observation of comparable duration (Ozawa et al. 2005). In addition to typical stellar flares, with a rapid rise followed by a slow decay, some sources show slower systematic variations, with some evidence for periodic variability, as observed also in other SFRs (Preibisch \& Zinnecker 2002; Skinner et al. 2003): such variations have been generally attributed to the effect of rotational modulation of active regions coming in and out of view as the star rotates (see, e.g. Stelzer et al. 1999).
Spectral analysis of the brightest sources indicates that the coronae of late-type stars in the $\sigma$ Ori cluster have typical temperatures of $0.3-0.8 \mathrm{keV}$ and $1-3 \mathrm{keV}$, with emission measure ratios of $E M_{2} / E M_{1} \sim 0.5-2.5$. In a few cases temperatures up to $7 \mathrm{keV}$ have been found even in the absence of clear flares. Our results are in very good agreement with the coronal temperatures generally found in SFRs (Feigelson \& Montmerle 1999; Imanishi et al. 2002; Favata et al. 2003; Preibisch \& Zinnecker 2004; Stelzer et al. 2004). In addition, we find for most stars a strongly subsolar metallicity $\left(Z \sim 0.1-0.3 Z_{\odot}\right)$, consistent with the results found in other SFRs (Imanishi et al. 2002; Favata et al. 2003; Ozawa et al. 2005), and in active latetype coronae.

A long debated question in the study of PMS stars is whether there is a difference between the X-ray properties of CTTS and WTTS, i.e. whether X-ray emission is influenced by the presence of an accretion disk. Observations of SFRs have shown no significant difference in the X-ray luminosity of CTTS and WTTS when using as a discriminant the presence of infrared excess, indicative of a circumstellar disk (Preibisch \& Zinnecker 2001; Getman et al. 2002; Feigelson et al. 2002, 2003; Gagné et al. 2004), while CTTS appear to be significantly less luminous than WTTS when using accretion indicators such as $\mathrm{H} \alpha$ or CaII emission (Stelzer \& Neuhäuser 2001; Preibisch \& Zinnecker 2002; Flaccomio et al. 2003c; Feigelson \& Lawson 2004; Stassun et al. 2004). The latter results are confirmed by our observations: using a selection in terms of $\mathrm{H} \alpha$ emission, we find in fact that the X-ray emission from CTTS is significantly weaker than WTTS.

For the small subsample of bright WTTS and CTTS for which we were able to perform spectral analysis, we find no significant difference in the temperatures and metallicity of the two classes. Both CTTS and WTTS show temperatures up to $\sim 3 \mathrm{keV}$, and metallicities $Z \sim 0.1-0.5 Z_{\odot}$. In L1551 Favata et al. (2003) found similar temperatures but a higher metallicity spread in CTTS with respect to WTTS, which is not confirmed by our data. We stress, however, that both their sample and ours are very small, preventing us to draw definite conclusions on possible abundance differences between the two classes. The high temperatures we find for CTTS are in constrast with the results obtained from high-resolution observations of TW Hya, whose corona shows a very low temperature $(T \sim 3 \mathrm{MK})$ and high density $\left(n_{\mathrm{e}} \gtrsim 10^{12} \mathrm{~cm}^{-3}\right)$ that have been interpreted as due to X-ray emission from an accretion shock (Kastner et al. 2002; Stelzer \& Schmitt 2004); however, other high-resolution observations of CTTS have shown the presence of hotter plasma (Schmitt et al. 2005; Argiroffi et al. 2005; Pallavicini et al. 2005), similar to what found in WTTS (Kastner et al. 2004; Argiroffi et al. 2004; Scelsi et al. 2005), suggesting a magnetic origin for X-ray emission.

Finally, we clearly detected 5 very low-mass objects and brown dwarf candidates down to M 7.5, with the possible detection of other two objects with ambiguous identification. X-ray luminosities range between $3 \times 10^{28}$ and $6 \times 10^{29} \mathrm{erg} \mathrm{s}^{-1}$, with $L_{\mathrm{X}} / L_{\mathrm{bol}} \sim 10^{-3.6}-10^{-2.5}$. Two of the candidate brown dwarfs (S Ori 3 and S Ori 25) show significant variability, while a flare is detected in SWW 203 (=NX 147), a very-low mass star of spectral type $\sim$ M 5 (Fig. 7). Several brown dwarfs and 
very-low mass stars have been detected in SFRs by ROSAT (Neuhäuser et al. 1999; Mokler \& Stelzer 2002), Chandra (Imanishi et al. 2001; Preibisch \& Zinnecker 2001; Feigelson et al. 2002; Gagné et al. 2004) and XMM-Newton (Stelzer et al. 2004; Ozawa et al. 2005), with $L_{X} \sim 10^{27}-10^{30} \mathrm{erg} \mathrm{s}^{-1}$ and $L_{\mathrm{X}} / L_{\mathrm{bol}} \sim 10^{-4.7}-10^{-2.6}$. Variability and flares, similar to those observed in late-type stars, have also been detected in several brown dwarfs. Our results are therefore consistent with previous observations.

Two of the very-low mass stars in our sample had been previously detected by ROSAT, i.e. SOri 3 and S Ori J053926.8-022614 (Mokler \& Stelzer 2002), with luminosities consistent with the ones derived here. We note however that S Ori J053926.8-022614 is very close to the $\sim \mathrm{M} 2$ cluster member SE 94, and its identification is ambiguous: from the XMM-Newton data it is not possible to determine which of the two stars is really responsible for the X-ray emission (see Sect. 2.2). At the time of the ROSAT observation SE 94 was not known, and the observed X-ray emission was attributed entirely to S Ori J053926.8-022614.

To conclude, the XMM-Newton observations of the cluster around $\sigma$ Ori reported in this paper are consistent with what is typically found in SFRs and very young clusters. The coronal temperatures, metallicities, $L_{\mathrm{X}} / L_{\mathrm{bol}}$ ratios and variability of the PMS stars in the $\sigma$ Ori cluster are similar to those typically observed in SFRs and clusters in the age range 1-4 Myr. The median X-ray luminosity and the detection rate of late-type stars in $\sigma$ Ori, however, are lower than in clusters of similar age, but this could be due to significant contamination by non-members. Although we found evidence of strong absorption in the spectra of some CTTS, and their X-ray luminosities are typically lower than those of WTTS, we do not find significant differences in the coronal temperatures and metallicities of CTTS with respect to WTTS. Our limited data sample does not support the view that CTTS as a class have all low coronal temperatures and high densities as found from high-resolution XMMNewton and Chandra observations of the CTT star TW Hya. While the latter behaviour has been interpreted as due to an accretion shock, our sample shows evidence for surface magnetic activity in at least some CTTS, similarly to what is typically observed in WTT stars.

Acknowledgements. We thank the anonymous referee for his/her comments, and E. Flaccomio for useful comments and discussions. E.F. and R.P. acknowledge partial support from Ministero dell'Istruzione, Università e Ricerca (MIUR). J.S.F. acknowledges support by the ESA Research Fellows program. This publication makes use of data products from the Two Micron All Sky Survey, which is a joint project of the University of Massachusetts and the Infrared Processing and Analysis Center/California Institute of Technology, and of the Guide Star Catalogue-II, which is a joint project of the Space Telescope Science Institute and INAFOsservatorio Astronomico di Torino.

\section{References}

Argiroffi, C., Drake, J. J., Maggio, A., et al. 2004, ApJ, 609, 925

Argiroffi, C., Maggio, A., Peres, G., Stelzer, B., \& Neuhäuser, R. 2005, A\&A, 439, 1149
Baraffe, I., Chabrier, G., Allard, F., \& Hauschildt, P. H. 1998, A\&A, 337,403

Barrado y Navascués, D., \& Martín, E. L. 2003, AJ, 126, 2997

Barrado y Navascués, D., Zapatero Osorio, M. R., Béjar, V. J. S., et al. 2001, A\&A, 377, L9

Barrado y Navascués, D., Béjar, V. J. S., Mundt, R., et al. 2003, A\&A, 404, 171

Béjar, V. J. S., Zapatero Osorio, M. R., \& Rebolo, R. 1999, ApJ, 521, 671

Béjar, V. J. S., Martín, E. L., Zapatero Osorio, M. R., et al. 2001, ApJ, 556,830

Béjar, V. J. S., Zapatero Osorio, M. R., \& Rebolo, R. 2004, AN, 325, 705

Berghöfer, T. W., Schmitt, J. H. M. M., Danner, R., \& Cassinelli, J. P. 1997, A\&A, 322, 167

Brown, A. G. A., de Geus, E. J., \& de Zeeuw, P. T. 1994, A\&A, 289, 101

Burningham, B., Naylor, T., Littlefair, S. P., \& Jeffries, R. D. 2005, MNRAS, 356, 1583

Caballero, J. A., Béjar, V. J. S., Rebolo, R., \& Zapatero Osorio, M. R. 2004, A\&A, 424, 857

Cutri, R. M., Skrutskie, M. F., van Dyk, S., et al. 2003, 2MASS AllSky Catalog of Point Sources

Damiani, F., Maggio, A., Micela, G., \& Sciortino, S. 1997, ApJ, 483, 350

ESA 1997, The Hipparcos and Tycho catalogues, ESA SP-1200

Favata, F., Giardino, G., Micela, G., Sciortino, S., \& Damiani, F. 2003 , A\&A, 403, 187

Feigelson, E. D., Broos, P., Gaffney, J. A., I., et al. 2002, ApJ, 574, 258

Feigelson, E. D., Gaffney, J. A., I., Garmire, G., Hillenbrand, L. A., \& Townsley, L. 2003, ApJ, 584, 911

Feigelson, E. D., \& Lawson, W. A. 2004, ApJ, 614, 267

Feigelson, E. D., \& Montmerle, T. 1999, ARA\&A, 37, 363

Flaccomio, E., Damiani, F., Micela, G., et al. 2003a, ApJ, 582, 382

Flaccomio, E., Damiani, F., Micela, G., et al. 2003b, ApJ, 582, 398

Flaccomio, E., Micela, G., \& Sciortino, S. 2003c, A\&A, 397, 611

Flaccomio, E., Micela, G., \& Sciortino, S. 2003d, A\&A, 402, 277

Gagné, M., Skinner, S. L., \& Daniel, K. J. 2004, ApJ, 613, 393

Garmire, G., Feigelson, E. D., Broos, P., et al. 2000, AJ, 120, 1426

Getman, K. V., Feigelson, E. D., Townsley, L., et al. 2002, ApJ, 575, 354

Hasinger, G., Altieri, B., Arnaud, M., et al. 2001, A\&A, 365, L45

Imanishi, K., Tsujimoto, M., \& Koyama, K. 2001, ApJ, 563, 361

Imanishi, K., Tsujimoto, M., \& Koyama, K. 2002, ApJ, 572, 300

Kastner, J. H., Huenemorder, D. P., Schulz, N. S., Canizares, C. R., \& Weintraub, D. A. 2002, ApJ, 567, 434

Kastner, J. H., Huenemorder, D. P., Schulz, N. S., et al. 2004, ApJ, 605, L49

Kenyon, M. J., Jeffries, R. D., Naylor, T., Oliveira, J. M., \& Maxted, P. F. L. 2005, MNRAS, 356, 89

Kenyon, S. J., \& Hartmann, L. 1995, ApJS, 101, 117

Lavalley, M., Isobe, T., \& Feigelson, E. 1992, in Astronomical Data Analysis Software and Systems, ed. D. M. Worrall et al., ASP Conf. Ser., 25, 245

Lee, T. A. 1968, ApJ, 152, 913

Leggett, S. K., Allard, F., Geballe, T. R., Hauschildt, P. H., \& Schweitzer, A. 2001, ApJ, 548, 908

Martín, E. L., Zapatero Osorio, M. R., Barrado y Navascués, D., Béjar, V. J. S., \& Rebolo, R. 2001, ApJ, 558, L117

Mokler, F., \& Stelzer, B. 2002, A\&A, 391, 1025

Muzerolle, J., Hillenbrand, L., Calvet, N., Briceño, C., \& Hartmann, L. 2003, ApJ, 266, 592 
Neuhäuser, R., Briceño, C., Comerón, F., et al. 1999, A\&A, 343, 883

Oliveira, J. M., Jeffries, R. D., Kenyon, M. J., Thompson, S. A., \& Naylor, T. 2002, A\&A, 382, L22

Oliveira, J. M., Jeffries, R. D., \& van Loon, J. T. 2004, MNRAS, 347, 1327

Oliveira, J. M., \& van Loon, J. T. 2004, A\&A, 418, 663

Ozawa, H., Grosso, N., \& Montmerle, T. 2005, A\&A, 429, 963

Pallavicini, R., Franciosini, E., Maggio, A., Scelsi, L., \& Sanz-Forcada, J. 2005, Adv. Sp. Res., in press

Pallavicini, R., Golub, L., Rosner, R., et al. 1981, ApJ, 248, 279

Preibisch, T., \& Zinnecker, H. 2001, AJ, 122, 866

Preibisch, T., \& Zinnecker, H. 2002, AJ, 123, 1613

Preibisch, T., \& Zinnecker, H. 2004, A\&A, 422, 1001

Ramírez, S. V., Rebull, L., Stauffer, J., et al. 2004, AJ, 127, 2659

Randich, S., \& Schmitt, J. H. M. M. 1995, A\&A, 298, 115

Sanz-Forcada, J., Franciosini, E., \& Pallavicini, R. 2004, A\&A, 421, 715

Scelsi, L., Maggio, A., Peres, G., \& Pallavicini, R. 2005, A\&A, 432, 671

Schmitt, J. H. M. M., Robrade, J., Ness, J.-U., Favata, F., \& Stelzer, B. 2005, A\&A, 432, L35
Scholz, A., \& Eislöffel, J. 2004, A\&A, 419, 249

Sherry, W. H., Walter, F. M., \& Wolk, S. J. 2004, AJ, 128, 2316

Siess, L., Dufour, E., \& Forestini, M. 2000, A\&A, 358, 593

Skinner, S., Gagné, M., \& Belzer, E. 2003, ApJ, 598, 375

Stassun, K. G., Ardila, D. R., Barsony, M., Basri, G., \& Mathieu, R. D. 2004, AJ, 127, 3537

Stelzer, B., Micela, G., \& Neuhäuser, R. 2004, A\&A, 423, 1029

Stelzer, B., \& Neuhäuser, R. 2001, A\&A, 377, 538

Stelzer, B., Neuhäuser, R., Casanova, S., \& Montmerle, T. 1999, A\&A, 344, 154

Stelzer, B., \& Schmitt, J. H. M. M. 2004, A\&A, 418, 687

Tozzi, P., Rosati, P., Nonino, M., et al. 2001, ApJ, 562, 42

Walter, F. M., Wolk, S. J., Freyberg, M., \& Schmitt, J. H. M. M. 1997, Mem. Soc. Astr. It., 68, 1081

Warren, W. H., J., \& Hesser, J. E. 1977, ApJS, 34, 115

Weaver, W. B., \& Babcock, A. 2004, PASP, 116, 1035

Wolk, S. J. 1996, Ph.D. Thesis, Univ. New York at Stony Brook

Zapatero Osorio, M. R., Béjar, V. J. S., Martín, E. L., et al. 2000, Science, 290, 103

Zapatero Osorio, M. R., Béjar, V. J. S., Pavlenko, Y., et al. 2002, A\&A, 384, 937 
E. Franciosini et al.: XMM-Newton observations of the $\sigma$ Orionis cluster. II., Online Material p 1

\section{Online Material}




\section{Appendix A: X-ray and optical properties of cluster members and candidates}

Table A.1. X-ray sources with at least one cluster member or candidate within 5". NX is a running identification number for the X-ray sources. The column labeled "Sign." indicates the significance of detection. Optical identifications are from the following sources: 4771-..., r05..., p05... = Wolk (1996); SE = Scholz \& Eislöffel (2004); SWW =Sherry et al. (2004); S Ori, J05... = Béjar et al. (1999, 2001, 2004); Zapatero Osorio et al. (2002); Caballero et al. (2004): note that we have dropped the S Ori prefix in front of the J05... names; K = Kenyon et al. (2005). The flag in the column labeled "C/W" indicates whether the star is a probable/possible CTTS or WTTS.

\begin{tabular}{|c|c|c|c|c|c|c|c|c|c|c|c|c|}
\hline NX & $\mathrm{RA}_{\mathrm{X}}$ & ${ }_{000)} \operatorname{Dec}_{X}$ & Sign. & $\begin{array}{r}\text { Count rate } \\
(\mathrm{cts} / \mathrm{ks})\end{array}$ & Optical ID & $\begin{array}{c}\Delta r \\
\left({ }^{\prime \prime}\right)\end{array}$ & $I$ & $R-I$ & $I-J$ & $\mathrm{SpT}^{a}$ & $\log L_{\mathrm{X}}^{b}$ & $\begin{array}{l}\mathrm{C} / \\
\mathrm{W}\end{array}$ \\
\hline 1 & $5: 37: 51.62$ & $-2: 35: 23.6$ & 21.5 & $10.55 \pm 0.94$ & SWW 125 & 2.48 & 13.34 & 1.22 & 1.45 & M2 & 30.02 & \\
\hline 2 & $5: 37: 53.03$ & $-2: 33: 33.7$ & 104.9 & $148.74 \pm 3.45$ & $4771-0775$ & 1.65 & 10.69 & 0.42 & 0.70 & K0* & $31.13^{\dagger}$ & $\mathrm{C}$ ? \\
\hline 3 & $5: 37: 54.44$ & $-2: 39: 27.8$ & 122.0 & $189.27 \pm 3.81$ & 4771-0921 & 2.22 & 10.04 & 0.51 & 0.78 & K0* & $31.24^{\dagger}$ & W? \\
\hline 4 & $5: 38: 00.85$ & $-2: 45: 09.4$ & 17.2 & $9.68 \pm 0.97$ & SWW 140 & 2.93 & 14.54 & 1.77 & 1.81 & M4 & 29.98 & \\
\hline 6 & $5: 38: 06.52$ & $-2: 28: 48.9$ & 5.4 & $0.67 \pm 0.18$ & $4771-0950$ & 1.11 & 10.70 & & 0.61 & F7 & 28.82 & $\mathrm{~W}$ ? \\
\hline 7 & $5: 38: 06.77$ & $-2: 30: 22.9$ & 50.8 & $19.05 \pm 0.86$ & SWW 113 & 1.02 & 13.14 & 0.94 & 1.38 & M0 & $30.77^{\dagger}$ & $\mathrm{C}$ \\
\hline 8 & 5:38:07.94 & $-2: 31: 31.0$ & 102.3 & $49.95 \pm 1.22$ & 4771-0854 & 0.38 & 11.45 & 0.64 & 0.88 & K4 & $30.66^{\dagger}$ & \\
\hline 9 & $5: 38: 08.33$ & $-2: 35: 57.5$ & 20.4 & $3.42 \pm 0.32$ & SWW 41 & 1.29 & 13.76 & 1.32 & 1.62 & M2 & 29.53 & W? \\
\hline 11 & $5: 38: 13.22$ & $-2: 26: 07.9$ & 9.1 & $1.63 \pm 0.29$ & SE 3 & 1.39 & 14.10 & 1.57 & 1.62 & M3 & 29.20 & \\
\hline 17 & $5: 38: 17.88$ & $-2: 40: 49.4$ & 9.1 & $0.97 \pm 0.17$ & J053817.8-024050 & 0.63 & 14.98 & 1.83 & 1.78 & M4 & 28.98 & \\
\hline 19 & $5: 38: 18.39$ & $-2: 35: 38.0$ & 9.9 & $0.95 \pm 0.16$ & J053818.2-023539 & 0.97 & 17.96 & 2.23 & 2.51 & M5 & 28.97 & \\
\hline 20 & $5: 38: 20.29$ & $-2: 38: 01.3$ & 15.7 & $1.37 \pm 0.18$ & J053820.1-023802 & 0.42 & 14.33 & 1.69 & 1.75 & M4.0* & 29.13 & $\mathrm{~W}$ \\
\hline 25 & $5: 38: 23.35$ & $-2: 44: 14.6$ & 8.6 & $0.85 \pm 0.17$ & J053823.3-024414 & 1.10 & 15.19 & 1.73 & 1.73 & M3 & 28.92 & \\
\hline 26 & $5: 38: 23.59$ & $-2: 41: 33.9$ & 9.1 & $0.96 \pm 0.19$ & J053823.6-024132 & 2.28 & 14.93 & 1.81 & 1.64 & M4 & 28.98 & \\
\hline 31 & $5: 38: 26.52$ & $-2: 34: 28.7$ & 31.7 & $4.68 \pm 0.29$ & $4771-1021$ & 0.46 & 12.10 & 0.50 & 0.68 & $\mathrm{~K} 2$ & 29.66 & \\
\hline 32 & $5: 38: 27.22$ & $-2: 45: 09.1$ & 24.2 & $4.76 \pm 0.38$ & $4771-0041$ & 2.03 & 12.82 & 0.82 & 0.87 & $\mathrm{~K} 7.0^{*}$ & 29.67 & $\mathrm{C}$ \\
\hline 33 & $5: 38: 27.62$ & $-2: 35: 02.6$ & 7.0 & $1.54 \pm 0.40$ & J053827.5-023504 & 1.60 & 14.41 & 1.42 & 1.58 & M3.5* & 29.18 & $\mathrm{C}$ \\
\hline 34 & $5: 38: 27.75$ & $-2: 43: 00.5$ & 39.7 & $8.69 \pm 0.49$ & SWW 87 & 1.36 & 13.67 & 1.33 & 1.48 & M3 & $29.96^{\dagger}$ & \\
\hline 39 & $5: 38: 29.19$ & $-2: 36: 02.7$ & 62.6 & $12.51 \pm 0.45$ & SWW 177 & 0.35 & 14.04 & 1.18 & 1.40 & M2 & $30.08^{\dagger}$ & \\
\hline 44 & $5: 38: 31.66$ & $-2: 35: 14.8$ & 28.3 & $3.89 \pm 0.29$ & r053831-0235 & 0.18 & 13.49 & 1.11 & 1.97 & M0.0* & 29.58 & $\mathrm{~W}$ \\
\hline 49 & $5: 38: 32.97$ & $-2: 35: 39.6$ & 74.7 & $18.73 \pm 0.54$ & $\mathrm{r} 053832-0235 \mathrm{~b}$ & 0.59 & 12.88 & 0.83 & 1.34 & $\mathrm{~K} 7$ & $30.27^{\dagger}$ & \\
\hline 52 & $5: 38: 33.36$ & $-2: 36: 17.6$ & 22.1 & $3.17 \pm 0.25$ & SWW 130 & 1.42 & 13.47 & 1.26 & 1.42 & M2 & 29.49 & \\
\hline 53 & $5: 38: 33.90$ & $-2: 44: 13.8$ & 30.9 & $7.72 \pm 0.55$ & $4771-1095$ & 1.84 & 11.47 & 0.81 & 1.34 & $\mathrm{~K} 5^{*}$ & 29.88 & $\mathrm{C}$ \\
\hline 54 & $5: 38: 34.03$ & $-2: 36: 37.5$ & 10.5 & $1.12 \pm 0.19$ & r053833-0236 & 1.84 & 13.72 & 1.52 & 1.74 & M3.5* & 29.04 & $\mathrm{C}$ \\
\hline 55 & $5: 38: 34.20$ & $-2: 34: 15.5$ & 39.7 & $5.86 \pm 0.35$ & HD 294272 & 4.54 & 8.57 & & & B9.5* & 29.76 & \\
\hline 60 & $5: 38: 35.74$ & $-2: 31: 52.2$ & 59.6 & $16.05 \pm 0.65$ & r053835-0231 & 2.83 & 12.45 & 0.79 & 1.15 & $\mathrm{~K} 5^{*}$ & 30.20 & $\mathrm{~W}$ \\
\hline 61 & $5: 38: 35.88$ & $-2: 43: 49.5$ & 40.3 & $9.16 \pm 0.48$ & 4771-1026 & 2.14 & 11.99 & 0.72 & 1.55 & $\mathrm{~K}^{*}$ & $30.38^{\dagger}$ & $\mathrm{C}$ \\
\hline 62 & $5: 38: 36.01$ & $-2: 30: 43.3$ & 209.2 & $143.97 \pm 1.82$ & $4771-1097$ & 0.67 & 12.47 & 0.81 & 1.23 & K6. $0^{*}$ & $31.08^{\dagger}$ & $\mathrm{W}$ \\
\hline 64 & $5: 38: 38.28$ & $-2: 36: 38.2$ & 123.3 & $37.57 \pm 0.74$ & r053838-0236 & 0.60 & 12.38 & 0.86 & 1.22 & K8.0* & $30.62^{\dagger}$ & $\mathrm{W}$ \\
\hline 65 & $5: 38: 38.54$ & $-2: 34: 55.5$ & 252.1 & $138.80 \pm 1.43$ & $4771-1147^{c}$ & 0.75 & 10.88 & 0.65 & 0.97 & $\mathrm{~K} 0^{*}$ & $31.07^{\dagger}$ & $\mathrm{W}$ \\
\hline \multirow[t]{2}{*}{67} & $5: 38: 39.13$ & $-2: 28: 00.4$ & 9.7 & $1.29 \pm 0.21$ & SE 70 & 2.60 & 16.99 & 1.78 & 1.72 & M4 & 29.11 & \\
\hline & & & & & S Ori 68 & 4.72 & 23.78 & & 3.60 & L5. $0^{*}$ & & $\mathrm{~W}$ \\
\hline 68 & $5: 38: 39.76$ & $-2: 40: 19.5$ & 10.4 & $0.98 \pm 0.15$ & p053839-0240 & 0.95 & 15.50 & 1.77 & 1.75 & M4 & 28.98 & \\
\hline 69 & $5: 38: 40.35$ & $-2: 30: 17.5$ & 62.6 & $16.11 \pm 0.57$ & r053840-0230 & 1.10 & 12.80 & 0.94 & 1.29 & M0.0* & $30.52^{\dagger}$ & $\mathrm{C}$ \\
\hline 70 & $5: 38: 41.34$ & $-2: 37: 22.3$ & 224.3 & $107.17 \pm 1.24$ & $\mathrm{r} 053841-0237^{d}$ & 0.78 & 12.77 & 0.87 & 1.31 & $\mathrm{~K} 3^{*}$ & $30.99^{\dagger}$ & $\mathrm{C}$ \\
\hline 71 & $5: 38: 41.46$ & $-2: 36: 43.7$ & 9.2 & $1.08 \pm 0.17$ & p053841-0236 & 0.81 & 14.52 & 1.36 & 1.53 & M3 & 29.03 & \\
\hline 75 & $5: 38: 43.38$ & $-2: 32: 00.5$ & 12.5 & $0.67 \pm 0.11$ & SWW 144 & 0.88 & 14.12 & 1.75 & 1.88 & M4 & 28.82 & \\
\hline 76 & $5: 38: 43.63$ & $-2: 33: 25.7$ & 83.1 & $19.35 \pm 0.55$ & SWW 36 & 0.38 & 13.09 & 1.06 & 1.37 & M1 & $30.28^{\dagger}$ & \\
\hline 78 & $5: 38: 44.27$ & $-2: 40: 19.7$ & 137.5 & $48.80 \pm 0.91$ & $4771-1051$ & 0.77 & 12.33 & 0.79 & 0.97 & $\mathrm{~K} 7.5^{*}$ & $30.68^{\dagger}$ & $\mathrm{W}$ \\
\hline 79 & $5: 38: 44.36$ & $-2: 32: 33.7$ & 162.5 & $64.62 \pm 1.02$ & $4771-1055$ & 0.34 & 12.04 & 0.86 & 1.16 & K8 & $30.81^{\dagger}$ & \\
\hline 80 & $5: 38: 44.86$ & $-2: 36: 00.2$ & 479.2 & $439.86 \pm 2.47$ & $\sigma$ Ori $\mathrm{AB}$ & 0.24 & 4.07 & & & O9.5* & $31.74^{\dagger}$ & \\
\hline 81 & $5: 38: 45.40$ & $-2: 41: 59.7$ & 72.1 & $19.21 \pm 0.62$ & SWW 97 & 1.00 & 13.46 & 1.16 & 1.47 & M1 & $30.38^{\dagger}$ & \\
\hline 82 & $5: 38: 46.10$ & $-2: 45: 25.1$ & 5.4 & $0.73 \pm 0.18$ & J053845.9-024523 & 1.98 & 15.52 & 1.84 & 1.96 & M4 & 28.86 & \\
\hline 84 & $5: 38: 47.26$ & $-2: 35: 39.9$ & 312.7 & $199.42 \pm 1.69$ & $\sigma$ Ori $\mathrm{E}$ & 0.81 & 6.49 & & & $\mathrm{~B} 2 \mathrm{p}^{*}$ & $31.46^{\dagger}$ & \\
\hline 87 & $5: 38: 47.56$ & $-2: 35: 24.7$ & 25.4 & $5.89 \pm 0.58$ & SWW 78 & 0.54 & 12.86 & 0.99 & 1.12 & M1 & 29.76 & \\
\hline 88 & $5: 38: 47.99$ & $-2: 37: 19.7$ & 25.0 & $3.99 \pm 0.26$ & SWW 102 & 0.55 & 13.02 & 1.18 & 1.00 & $\mathrm{~K} 5^{*}$ & 29.59 & $\mathrm{~W}$ \\
\hline 89 & $5: 38: 48.05$ & $-2: 30: 39.3$ & 5.5 & $2.15 \pm 0.54$ & S Ori $6^{e}$ & 4.88 & 15.53 & 2.00 & 1.88 & M5 & 28.83 & \\
\hline 90 & $5: 38: 48.18$ & $-2: 27: 13.3$ & 166.7 & $106.85 \pm 1.72$ & 4771-0899 & 1.14 & 11.35 & 0.82 & 1.19 & K7.0* & $31.21^{\dagger}$ & $\mathrm{W}$ \\
\hline 92 & $5: 38: 48.76$ & $-2: 36: 16.7$ & 59.5 & $13.60 \pm 0.48$ & SWW 35 & 0.51 & 13.37 & 0.87 & 1.26 & M1 & $30.20^{\dagger}$ & \\
\hline 93 & $5: 38: 49.26$ & $-2: 38: 23.5$ & 93.0 & $22.49 \pm 0.58$ & r053849-0238 & 1.28 & 12.96 & 1.03 & 1.57 & M0.5* & $30.31^{\dagger}$ & $\mathrm{C}$ \\
\hline 94 & 5:38:49.27 & $-2: 41: 25.3$ & 13.9 & $1.00 \pm 0.16$ & SWW 205 & 0.78 & 13.23 & 1.50 & 1.56 & M3 & 28.99 & \\
\hline
\end{tabular}


Table A.1. continued.

\begin{tabular}{|c|c|c|c|c|c|c|c|c|c|c|c|c|}
\hline NX & $\mathrm{RA}_{\mathrm{X}}$ & $00)^{\operatorname{Dec}_{X}}$ & Sign. & $\begin{array}{r}\text { Count rate } \\
(\mathrm{cts} / \mathrm{ks})\end{array}$ & Optical ID & $\begin{array}{c}\Delta r \\
\left({ }^{\prime \prime}\right)\end{array}$ & $I$ & $R-I$ & $I-J$ & $\mathrm{SpT}^{a}$ & $\log L_{\mathrm{X}}^{b}$ & $\begin{array}{l}\mathrm{C} / \\
\mathrm{W}\end{array}$ \\
\hline 97 & $5: 38: 49.90$ & $-2: 41: 23.4$ & 15.2 & $1.08 \pm 0.15$ & SWW 200 & 0.75 & 14.21 & 1.37 & 1.46 & M3 & 29.03 & \\
\hline 98 & $5: 38: 50.19$ & $-2: 37: 34.4$ & 28.0 & $3.68 \pm 0.25$ & SWW 18 & 1.38 & 14.83 & 1.69 & 1.78 & M4 & 29.56 & \\
\hline 100 & $5: 38: 50.54$ & $-2: 26: 47.0$ & 7.6 & $1.04 \pm 0.23$ & SE 34 & 1.08 & 14.10 & 1.48 & 1.60 & M3 & 29.01 & \\
\hline 102 & $5: 38: 51.53$ & $-2: 36: 20.7$ & 39.7 & $5.03 \pm 0.29$ & r053851-0236 & 0.25 & 13.38 & 0.87 & 0.94 & K9 & 29.69 & $\mathrm{C}$ \\
\hline 104 & $5: 38: 52.07$ & $-2: 46: 43.9$ & 52.2 & $18.84 \pm 0.79$ & 4771-0080 & 0.57 & 12.78 & 0.80 & 1.26 & K7 & $30.19^{\dagger}$ & W \\
\hline 105 & $5: 38: 53.15$ & $-2: 38: 53.1$ & 17.6 & $1.72 \pm 0.18$ & SWW 166 & 0.57 & 12.78 & 1.07 & 1.16 & M1 & 29.23 & \\
\hline 106 & $5: 38: 53.20$ & $-2: 43: 53.5$ & 55.5 & $15.01 \pm 0.60$ & SWW 47 & 1.24 & 13.49 & 1.08 & 1.26 & M1 & $30.12^{\dagger}$ & W \\
\hline 109 & $5: 38: 53.63$ & $-2: 33: 23.0$ & 159.2 & $69.21 \pm 1.20$ & 4771-1049 & 2.39 & 11.57 & 0.73 & 0.96 & K5* & $30.86^{\dagger}$ & W \\
\hline 110 & $5: 38: 54.19$ & $-2: 49: 29.4$ & 50.8 & $53.52 \pm 2.31$ & 4771-0119 & 0.41 & 11.73 & 0.58 & 0.90 & $\mathrm{~K} 2^{*}$ & $30.67^{\dagger}$ & $\mathrm{W}$ \\
\hline 111 & $5: 38: 55.13$ & $-2: 28: 58.9$ & 5.9 & $0.69 \pm 0.16$ & SE 77 & 1.79 & 15.50 & 1.76 & 1.70 & M4 & 28.83 & \\
\hline 117 & $5: 38: 59.23$ & $-2: 47: 14.1$ & 23.7 & $5.83 \pm 0.48$ & r053859-0247 & 0.82 & 12.36 & 0.69 & 1.04 & K5 & 29.76 & \\
\hline 118 & $5: 38: 59.32$ & $-2: 33: 49.3$ & 6.8 & $0.25 \pm 0.07$ & SWW 227 & 2.09 & 14.83 & 1.26 & 1.94 & M0 & 28.39 & $\mathrm{C} ?$ \\
\hline 122 & 5:39:00.65 & $-2: 39: 39.9$ & 49.5 & $9.95 \pm 0.44$ & 4771-1056 & 1.01 & 12.43 & 0.49 & 0.77 & $\mathrm{~K} 1^{*}$ & $29.97^{\dagger}$ & $\mathrm{W}$ ? \\
\hline 124 & 5:39:01.24 & $-2: 36: 39.2$ & 11.1 & $0.80 \pm 0.12$ & K 9 & 0.38 & 15.11 & 1.56 & 1.59 & M3 & 28.90 & \\
\hline 126 & 5:39:02.89 & $-2: 29: 55.7$ & 30.2 & $5.32 \pm 0.36$ & SWW 28 & 0.53 & 14.21 & 1.51 & 1.60 & M3 & 29.72 & \\
\hline 131 & 5:39:05.30 & $-2: 33: 00.2$ & 7.2 & $0.34 \pm 0.09$ & SWW 175 & 0.64 & 15.02 & 1.55 & 1.63 & M3 & 28.52 & \\
\hline 132 & 5:39:05.49 & $-2: 32: 30.7$ & 52.2 & $12.41 \pm 0.52$ & $4771-1075$ & 0.40 & 12.66 & 0.87 & 1.11 & $\mathrm{~K} 7.0^{*}$ & $30.10^{\dagger}$ & $\mathrm{W}$ \\
\hline 137 & 5:39:07.69 & $-2: 28: 21.5$ & 11.5 & $1.76 \pm 0.26$ & r053907-0228 & 1.92 & 14.37 & 1.45 & 1.49 & M3.0* & 29.24 & $\mathrm{~W}$ \\
\hline 138 & 5:39:07.77 & $-2: 32: 39.7$ & 56.0 & $19.63 \pm 0.83$ & 4771-1092 & 1.24 & 12.63 & 0.81 & 1.33 & K5 ${ }^{*}$ & $30.50^{\dagger}$ & $\mathrm{C}$ \\
\hline 140 & 5:39:09.21 & $-2: 39: 59.4$ & 5.0 & $0.31 \pm 0.09$ & S Ori 25 & 2.87 & 17.16 & 2.17 & 2.27 & M7.5* & 28.48 & $\mathrm{C} ?$ \\
\hline 144 & $5: 39: 11.66$ & $-2: 31: 06.0$ & 26.6 & $13.68 \pm 0.93$ & SWW 195 & 0.98 & 13.45 & 0.95 & 1.46 & $\mathrm{~K} 5^{*}$ & 30.13 & $\mathrm{C}$ \\
\hline 145 & $5: 39: 11.74$ & $-2: 36: 03.3$ & 60.8 & $15.85 \pm 0.59$ & $4771-1038$ & 0.54 & 12.93 & 0.95 & 1.31 & $\mathrm{~K} 8^{*}$ & $30.32^{\dagger}$ & $\mathrm{W}$ \\
\hline 147 & $5: 39: 12.74$ & $-2: 30: 07.7$ & 12.0 & $2.09 \pm 0.36$ & SWW 203 & 4.95 & 14.69 & 2.00 & 2.08 & M5 & 29.31 & \\
\hline 149 & $5: 39: 14.60$ & $-2: 28: 33.4$ & 36.5 & $10.90 \pm 0.62$ & J053914.5-022834 & 0.55 & 14.85 & 1.52 & 1.51 & M3.5* & $30.17^{\dagger}$ & $\mathrm{W}$ \\
\hline 150 & $5: 39: 15.18$ & $-2: 31: 37.9$ & 39.4 & $9.86 \pm 0.53$ & HD 37564 & 0.52 & 8.17 & & 0.19 & $\mathrm{~A} 8^{*}$ & $29.94^{\dagger}$ & \\
\hline 154 & $5: 39: 17.41$ & $-2: 25: 44.2$ & 7.7 & $1.68 \pm 0.33$ & SE 51 & 2.28 & 14.18 & 1.15 & 1.28 & M2 & 29.22 & $\mathrm{C} ?$ \\
\hline 156 & $5: 39: 18.22$ & $-2: 29: 28.7$ & 58.9 & $23.27 \pm 0.90$ & $4771-0598$ & 0.85 & 11.41 & 0.47 & 0.69 & $\mathrm{~K} 1$ & $30.28^{\dagger}$ & \\
\hline 157 & 5:39:18.97 & $-2: 30: 55.6$ & 15.1 & $2.98 \pm 0.38$ & 4771-0910 & 2.53 & 12.58 & 0.84 & 1.18 & K3* & 29.47 & $\mathrm{C}$ \\
\hline 159 & $5: 39: 20.62$ & $-2: 27: 37.8$ & 26.1 & $7.93 \pm 0.60$ & J053920.5-022737 & 1.58 & 13.52 & 1.29 & 1.37 & M2.0* & 29.89 & $\mathrm{~W}$ \\
\hline 160 & $5: 39: 21.08$ & $-2: 30: 33.8$ & 24.3 & $5.77 \pm 0.46$ & S Ori 3 & 0.44 & 15.40 & 1.98 & 2.11 & M6 & 29.75 & \\
\hline 161 & $5: 39: 23.02$ & $-2: 33: 34.3$ & 23.5 & $4.69 \pm 0.39$ & r053923-0233 & 1.56 & 14.19 & 1.21 & 1.36 & M2.0* & 29.66 & $\mathrm{~W}$ \\
\hline 164 & $5: 39: 24.48$ & $-2: 34: 02.7$ & 16.9 & $3.35 \pm 0.36$ & SWW 127 & 1.39 & 14.28 & 1.25 & 1.30 & M2 & 29.52 & \\
\hline 165 & $5: 39: 25.33$ & $-2: 38: 24.3$ & 11.1 & $1.37 \pm 0.22$ & SWW 135 & 2.34 & 12.83 & 0.95 & 1.52 & M0 & 29.13 & $\mathrm{C} ?$ \\
\hline \multirow[t]{2}{*}{167} & $5: 39: 26.73$ & $-2: 26: 17.4$ & 9.6 & $2.94 \pm 0.56$ & SE 94 & 3.18 & 14.81 & 1.17 & 2.37 & M2 & 29.46 & \\
\hline & & & & & J053926.8-0226 & 3.92 & 18.66 & & 1.41 & M6 & & \\
\hline 170 & $5: 39: 32.68$ & $-2: 39: 45.2$ & 42.8 & $16.59 \pm 0.85$ & r053932-0239 & 1.23 & 12.23 & 0.81 & 1.41 & M0 & $30.19^{\dagger}$ & \\
\hline 172 & $5: 39: 36.77$ & $-2: 42: 17.4$ & 72.8 & $63.33 \pm 2.16$ & r053936-0242 & 1.99 & 9.11 & 0.40 & 0.65 & $\mathrm{G}^{*}$ & $30.96^{\dagger}$ & $\mathrm{W}$ \\
\hline 173 & $5: 39: 37.28$ & $-2: 26: 54.7$ & 10.7 & $4.40 \pm 0.75$ & SWW 163 & 2.62 & 12.98 & 0.91 & 1.28 & M0 & 29.64 & \\
\hline 174 & $5: 39: 39.96$ & $-2: 33: 16.8$ & 40.3 & $19.85 \pm 1.04$ & V603 Ori & 1.01 & 14.40 & & 2.18 & M2 & $31.09^{\dagger}$ & $\mathrm{C}$ \\
\hline
\end{tabular}

${ }^{a}$ Spectroscopically-determined spectral types from the literature are marked with an asterisk. The other spectral types have been estimated from the $R-I, V-I$ or $I-J$ colours using the relations by Kenyon \& Hartmann (1995) and Leggett et al. (2001).

${ }^{b}$ Unabsorbed X-ray luminosity in the $0.3-8 \mathrm{keV}$ band. Values marked with a $†$ have been derived from the PN or MOS 1 spectral fits reported in Table 3 and in Paper I.

${ }^{c}$ Source \#3 in Paper I.

${ }^{d}$ Source \#4 in Paper I.

${ }^{e}$ Source detected on the PN only. The reported count rates are PN count rates. $L_{\mathrm{X}}$ was derived using the conversion factor for PN (see Sect. 3.1). 
Table A.2. $3 \sigma$ upper limits for undetected late-type cluster members or candidates. Optical identifications are from the same sources as in Table A.1, with the addition of two stars from Burningham et al. (2005,B).

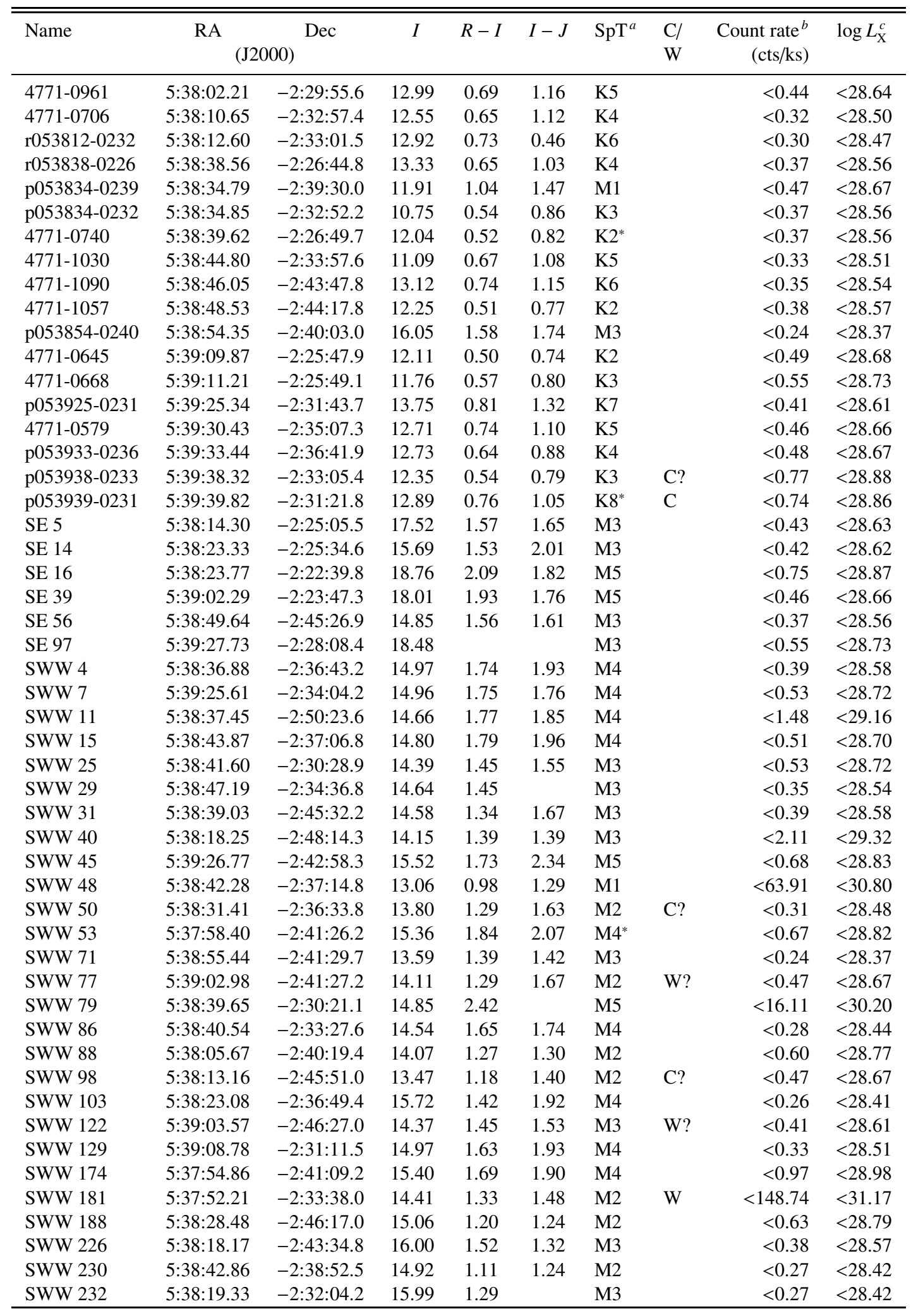


E. Franciosini et al.: XMM-Newton observations of the $\sigma$ Orionis cluster. II., Online Material p 5

Table A.2. continued.

\begin{tabular}{|c|c|c|c|c|c|c|c|c|c|}
\hline \multicolumn{6}{|c|}{ (J2000) } & $\mathrm{SpT}^{a}$ & $\begin{array}{l}\mathrm{C} / \\
\mathrm{W}\end{array}$ & $\begin{array}{r}\text { Count rate } \\
(\mathrm{cts} / \mathrm{ks})\end{array}$ & $\log L_{\mathrm{X}}^{c}$ \\
\hline S Ori 1 & $5: 39: 11.83$ & $-2: 27: 41.0$ & 15.08 & 1.70 & 1.47 & M4 & & $<0.78$ & $<28.89$ \\
\hline S Ori 2 & $5: 39: 26.33$ & $-2: 28: 37.7$ & 15.26 & 1.80 & 1.76 & M4 & & $<0.57$ & $<28.75$ \\
\hline S Ori 4 & $5: 39: 39.32$ & $-2: 32: 25.2$ & 15.23 & 2.16 & 1.79 & M5 & & $<0.70$ & $<28.84$ \\
\hline S Ori 5 & $5: 39: 20.24$ & $-2: 38: 25.9$ & 15.40 & 1.86 & 1.79 & M5 & & $<0.34$ & $<28.52$ \\
\hline S Ori 7 & 5:39:08.22 & $-2: 32: 28.4$ & 15.63 & 2.07 & 1.83 & M4-5 & & $<0.44$ & $<28.64$ \\
\hline S Ori 8 & 5:39:08.09 & $-2: 28: 44.8$ & 15.74 & 1.87 & 1.60 & M4 & & $<0.49$ & $<28.68$ \\
\hline S Ori 11 & $5: 39: 44.33$ & $-2: 33: 02.8$ & 16.39 & 2.04 & 2.10 & M6.0* & W? & $<0.98$ & $<28.98$ \\
\hline S Ori 12 & $5: 37: 57.46$ & $-2: 38: 44.4$ & 16.28 & 1.87 & 2.05 & M6.0* & W? & $<0.74$ & $<28.86$ \\
\hline S Ori 14 & $5: 39: 37.60$ & $-2: 44: 30.5$ & 16.75 & 1.94 & 2.37 & M6 & & $<0.86$ & $<28.93$ \\
\hline S Ori 15 & $5: 38: 48.10$ & $-2: 28: 53.6$ & 16.39 & 1.97 & 1.92 & M5.5* & W? & $<0.38$ & $<28.57$ \\
\hline S Ori 16 & $5: 39: 15.10$ & $-2: 40: 47.6$ & 16.62 & 1.91 & 1.95 & M5 & & $<0.30$ & $<28.47$ \\
\hline S Ori 17 & 5:39:04.49 & $-2: 38: 35.4$ & 16.95 & 1.88 & 2.18 & M6.0* & W? & $<0.28$ & $<28.44$ \\
\hline S Ori 18 & $5: 38: 25.68$ & $-2: 31: 21.7$ & 16.61 & 1.91 & 1.94 & M5 & & $<0.27$ & $<28.42$ \\
\hline S Ori 21 & $5: 39: 34.33$ & $-2: 38: 46.9$ & 17.25 & 2.02 & 2.43 & M6 & & $<0.50$ & $<28.69$ \\
\hline S Ori 22 & $5: 38: 35.36$ & $-2: 25: 22.2$ & 16.88 & 2.04 & 2.24 & M6.0* & W? & $<0.49$ & $<28.68$ \\
\hline S Ori 27 & $5: 38: 17.42$ & $-2: 40: 24.3$ & 17.03 & 2.16 & 2.20 & M7.0* & W & $<0$ & $<28.47$ \\
\hline S Ori 28 & $5: 39: 23.19$ & $-2: 46: 55.8$ & 17.14 & 2.05 & 1.81 & M5 & & $<0.71$ & $<28.84$ \\
\hline S Ori 29 & $5: 38: 29.61$ & $-2: 25: 14.2$ & 17.02 & 2.00 & 2.18 & M6. $5^{*}$ & $\mathrm{C} ?$ & $<0.41$ & $<28.61$ \\
\hline S Ori 30 & $5: 39: 13.08$ & $-2: 37: 50.9$ & 17.29 & 1.71 & 2.05 & M6 ${ }^{*}$ & W? & $<1.18$ & $<29.06$ \\
\hline S Ori 31 & $5: 38: 20.88$ & $-2: 46: 13.3$ & 17.23 & 2.35 & 2.04 & M7.0* & $\mathrm{W}$ ? & $<0.51$ & $<28.70$ \\
\hline S Ori 35 & $5: 37: 55.60$ & $-2: 33: 05.3$ & 17.61 & 2.25 & 2.39 & M6 & & $<0.99$ & $<28.99$ \\
\hline S Ori 36 & $5: 39: 26.85$ & $-2: 36: 56.2$ & 17.71 & 1.88 & 2.25 & M6 & & $<0.40$ & $<28.59$ \\
\hline S Ori 39 & $5: 38: 32.44$ & $-2: 29: 57.3$ & 17.67 & 2.09 & 2.23 & M6.5* & $\mathrm{W}$ ? & $<0.31$ & $<28.48$ \\
\hline S Ori 41 & $5: 39: 38.49$ & $-2: 31: 13.1$ & 18.44 & 2.43 & 1.73 & M6 & & $<0.65$ & $<28.81$ \\
\hline S Ori 42 & $5: 39: 23.41$ & $-2: 40: 57.5$ & 19.04 & 2.47 & 2.31 & M7. $5^{*}$ & $\mathrm{C}$ & $<0.37$ & $<28.56$ \\
\hline S Ori 43 & $5: 38: 13.95$ & $-2: 35: 01.5$ & 19.05 & 2.38 & & M7 & & $<3.71$ & $<29.56$ \\
\hline S Ori 44 & $5: 38: 07.13$ & $-2: 43: 21.0$ & 19.39 & 2.31 & 2.16 & M7.0* & $\mathrm{W}$ ? & $<0.41$ & $<28.61$ \\
\hline S Ori 47 & $5: 38: 14.62$ & $-2: 40: 15.4$ & 20.49 & 2.40 & 2.96 & $\mathrm{~L} 1.5^{*}$ & $\mathrm{C} ?$ & $<0.28$ & $<28.44$ \\
\hline S Ori 50 & $5: 39: 10.80$ & $-2: 37: 15.0$ & 20.48 & & 2.88 & M9.0* & W & $<0.40$ & $<28.60$ \\
\hline S Ori 51 & 5:39:03.20 & $-2: 30: 20.0$ & 20.23 & & 2.85 & M9.0* & $\mathrm{C} ?$ & & $<28.54$ \\
\hline S Ori 53 & $5: 38: 25.10$ & $-2: 48: 03.0$ & 21.17 & & 3.28 & M9.0* & $\mathrm{W}$ & $<0.76$ & $<28.87$ \\
\hline S Ori 58 & 5:39:03.60 & $-2: 25: 36.0$ & 21.90 & & 3.30 & L0. $0^{*}$ & $\mathrm{C} ?$ & $<0.42$ & $<28.62$ \\
\hline S Ori 60 & $5: 39: 37.50$ & $-2: 30: 42.0$ & 22.76 & & 3.62 & L2.0* & $\mathrm{C} ?$ & $<0.77$ & $<28.88$ \\
\hline S Ori 62 & $5: 39: 42.10$ & $-2: 30: 31.0$ & 23.03 & & 3.58 & L2.0* & $\mathrm{C} ?$ & $<0$ & $<28.93$ \\
\hline S Ori 65 & $5: 38: 26.10$ & $-2: 23: 05.0$ & 23.24 & & 3.34 & L3.5* & $\mathrm{W}$ & $<0.51$ & $<28.70$ \\
\hline S Ori 69 & $5: 39: 18.10$ & $-2: 28: 55.0$ & 23.89 & & 3.63 & $\mathrm{~T} 0^{*}$ & & $<0.56$ & $<28.74$ \\
\hline S Ori 70 & $5: 38: 10.10$ & $-2: 36: 26.0$ & 25.03 & & 4.75 & $\mathrm{~T} 5.5^{*}$ & & $<0.34$ & $<28.52$ \\
\hline S Ori 71 & $5: 39: 00.20$ & $-2: 37: 06.0$ & 20.02 & & 2.69 & $\mathrm{LO}^{*}$ & $\mathrm{C}$ & $<0.24$ & $<28.37$ \\
\hline J053805.5-023557 & $5: 38: 05.52$ & $-2: 35: 57.1$ & 17.66 & 2.10 & 2.38 & M6 & & $<0.36$ & $<28.55$ \\
\hline J053811.9-024557 & $5: 38: 11.90$ & $-2: 45: 56.8$ & 15.72 & 1.87 & 1.80 & M5 & & $<0.48$ & $<28.67$ \\
\hline J053816.0-023805 & $5: 38: 16.10$ & $-2: 38: 04.9$ & 15.19 & 1.57 & 1.61 & M4 & & $<0.81$ & $<28.90$ \\
\hline J053820.5-023409 & $5: 38: 20.50$ & $-2: 34: 09.0$ & 14.36 & 1.71 & 1.71 & M4.0* & $\mathrm{C} ?$ & $<0.33$ & $<28.51$ \\
\hline J053821.3-023336 & $5: 38: 21.38$ & $-2: 33: 36.3$ & 17.58 & 2.13 & 2.22 & M5 & & $<0.31$ & $<28.48$ \\
\hline J053825.4-024241 & $5: 38: 25.43$ & $-2: 42: 41.3$ & 16.86 & 1.80 & 1.98 & M4 & & $<0.34$ & $<28.52$ \\
\hline J053826.2-024041 & $5: 38: 26.23$ & $-2: 40: 41.3$ & 16.93 & 2.13 & 2.02 & M8.0* & $\mathrm{W}$ ? & $<0.24$ & $<28.37$ \\
\hline J053826.8-022846 & $5: 38: 26.84$ & $-2: 38: 46.0$ & 16.12 & 1.90 & 2.01 & M5 & & $<0.25$ & $<28.39$ \\
\hline J053829.0-024847 & $5: 38: 28.97$ & $-2: 48: 47.3$ & 16.81 & & 1.99 & M6 & W? & $<0.72$ & $<28.85$ \\
\hline J053833.9-024508 & $5: 38: 33.88$ & $-2: 45: 07.8$ & 15.98 & 1.89 & 1.73 & M4 & & $<0.61$ & $<28.78$ \\
\hline J053834.5-024109 & $5: 38: 34.60$ & $-2: 41: 08.8$ & 14.72 & 1.46 & 1.62 & M4 & & $<0.30$ & $<28.47$ \\
\hline J053836.7-024414 & $5: 38: 36.69$ & $-2: 44: 13.7$ & 14.32 & 1.76 & 1.78 & M4 & & $<0.45$ & $<28.65$ \\
\hline J053838.6-024157 & $5: 38: 38.59$ & $-2: 41: 55.9$ & 16.52 & 1.82 & 1.96 & M5 & & $<0.27$ & $<28.42$ \\
\hline J053844.4-024037 & $5: 38: 44.48$ & $-2: 40: 37.6$ & 17.28 & 2.32 & 2.48 & M6 & & $<0.44$ & $<28.64$ \\
\hline J053844.4-024030 & $5: 38: 44.49$ & $-2: 40: 30.5$ & 15.02 & 1.49 & 1.66 & M3 & & $<48.79$ & $<30.68$ \\
\hline
\end{tabular}


Table A.2. continued.

\begin{tabular}{|c|c|c|c|c|c|c|c|c|c|}
\hline \multicolumn{7}{|c|}{ (J2000) } & $\begin{array}{l}\mathrm{C} / \\
\mathrm{W}\end{array}$ & $\begin{array}{r}\text { Count rate }^{b} \\
(\mathrm{cts} / \mathrm{ks})\end{array}$ & $\log L_{\mathrm{X}}^{c}$ \\
\hline J053847.5-022711 & $5: 38: 47.54$ & $-2: 27: 12.0$ & 14.46 & 1.74 & & M5.0* & $\mathrm{W}$ & $<106.85$ & $<31.02$ \\
\hline J053848.1-022401 & $5: 38: 48.19$ & $-2: 44: 00.8$ & 16.13 & 1.91 & 2.06 & M5 & & $<0.38$ & $<28.57$ \\
\hline J053849.2-022358 & $5: 38: 49.29$ & $-2: 23: 57.6$ & 16.27 & 1.74 & 1.91 & M4.0* & $\mathrm{C}$ & $<0.45$ & $<28.65$ \\
\hline J053849.5-024934 & $5: 38: 49.50$ & $-2: 49: 34.0$ & 20.08 & & 2.80 & M7 & & $<0.95$ & $<28.97$ \\
\hline J053850.6-024244 & $5: 38: 50.61$ & $-2: 42: 42.9$ & 15.84 & 1.85 & 2.00 & M5 & & $<0.29$ & $<28.46$ \\
\hline J053853.8-024459 & $5: 38: 53.82$ & $-2: 44: 58.9$ & 17.78 & 2.08 & 2.33 & M6 & & $<0.33$ & $<28.51$ \\
\hline J053902.1-023501 & $5: 39: 01.94$ & $-2: 35: 02.9$ & 16.41 & & 1.96 & M4 & & $<0.25$ & $<28.39$ \\
\hline J053909.9-022814 & $5: 39: 10.01$ & $-2: 28: 11.6$ & 16.48 & 1.63 & 1.88 & M5.0* & $\mathrm{W} ?$ & $<0.41$ & $<28.61$ \\
\hline J053911.4-023333 & $5: 39: 11.40$ & $-2: 33: 32.8$ & 16.42 & 1.89 & 1.97 & M5.0* & W? & $<0.45$ & $<28.65$ \\
\hline J053912.8-022453 & $5: 39: 12.89$ & $-2: 24: 53.8$ & 19.42 & & 2.61 & M6.0* & $\mathrm{W}$ & $<1.53$ & $<29.18$ \\
\hline J053922.2-024552 & $5: 39: 22.25$ & $-2: 45: 52.4$ & 17.05 & & 1.73 & M4 & & $<0.50$ & $<28.69$ \\
\hline J053929.4-024636 & $5: 39: 29.40$ & $-2: 46: 36.0$ & 19.73 & & 2.55 & M6 & & $<0.72$ & $<28.85$ \\
\hline J053936.4-023626 & $5: 39: 36.40$ & $-2: 36: 26.0$ & 18.46 & & 2.52 & M6 & & $<0.52$ & $<28.71$ \\
\hline J053943.2-022343 & $5: 39: 43.19$ & $-2: 32: 43.3$ & 14.70 & 1.64 & 1.67 & M4 & & $<1.32$ & $<29.11$ \\
\hline K 8 & $5: 38: 50.78$ & $-2: 36: 26.8$ & 15.06 & 2.00 & 1.95 & M4 & & $<4.98$ & $<29.69$ \\
\hline K 50 & $5: 38: 51.00$ & $-2: 49: 14.0$ & 16.79 & 1.79 & 1.75 & M4 & & $<0.98$ & $<28.98$ \\
\hline K 62 & $5: 37: 52.07$ & $-2: 36: 04.7$ & 17.30 & 1.91 & 2.16 & M5 & & $<0.70$ & $<28.84$ \\
\hline K 65 & $5: 38: 39.76$ & $-2: 32: 20.3$ & 17.58 & 1.89 & 2.69 & M5 & & $<0.30$ & $<28.47$ \\
\hline B $3.01-67$ & $5: 38: 46.85$ & $-2: 36: 43.5$ & 15.28 & 1.88 & 2.06 & M5 & & $<0.53$ & $<28.72$ \\
\hline B $2.03-233$ & $5: 39: 40.58$ & $-2: 39: 12.3$ & 17.29 & 1.60 & 1.89 & M4 & & $<0.74$ & $<28.86$ \\
\hline
\end{tabular}

${ }^{a}$ See note to Table A.1.

${ }^{b}$ Upper limits higher than $10 \mathrm{cts} / \mathrm{ks}$ are due to the presence of a very close bright X-ray source and are therefore overestimated.

${ }^{c}$ Unabsorbed X-ray luminosity in the $0.3-8 \mathrm{keV}$ band. 
E. Franciosini et al.: XMM-Newton observations of the $\sigma$ Orionis cluster. II., Online Material $p 7$

\section{Appendix B: Unidentified X-ray sources}

Table B.1. X-ray sources with no known counterpart within $5^{\prime \prime}$. Sources NX $=59$ and 130 have been detected on one MOS only. The column labeled "Sign." indicates the significance of detection.

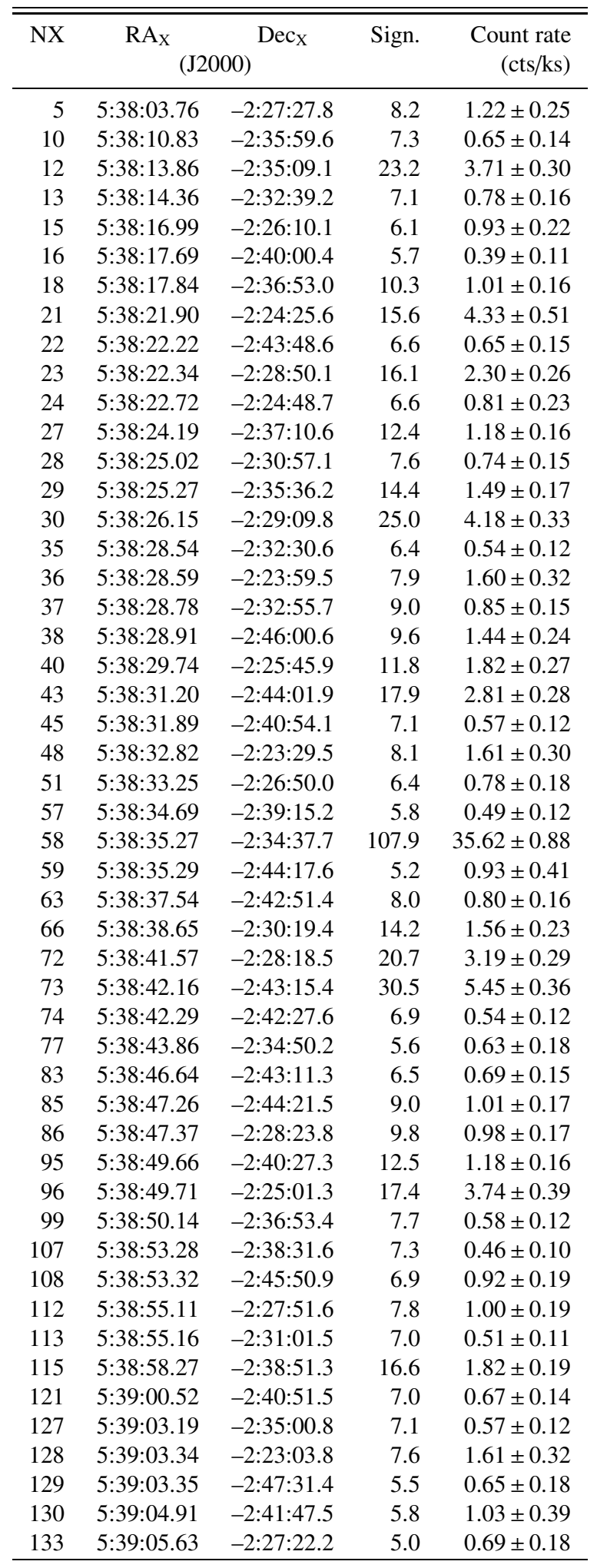

Table B.1. continued.

\begin{tabular}{cccrr}
\hline \hline NX & RA $_{X}$ & Dec $_{X}$ & Sign. & $\begin{array}{r}\text { Count rate } \\
(\mathrm{cts} / \mathrm{ks})\end{array}$ \\
\hline 134 & $5: 39: 05.72$ & $-2: 43: 16.6$ & 6.4 & $0.54 \pm 0.15$ \\
135 & $5: 39: 06.90$ & $-2: 30: 49.7$ & 6.7 & $0.72 \pm 0.18$ \\
136 & $5: 39: 07.05$ & $-2: 33: 41.5$ & 12.5 & $1.31 \pm 0.24$ \\
139 & $5: 39: 08.52$ & $-2: 44: 22.3$ & 5.7 & $0.61 \pm 0.15$ \\
141 & $5: 39: 09.56$ & $-2: 23: 40.9$ & 6.7 & $0.97 \pm 0.29$ \\
142 & $5: 39: 10.94$ & $-2: 26: 35.5$ & 7.1 & $1.23 \pm 0.26$ \\
143 & $5: 39: 11.13$ & $-2: 36: 46.6$ & 6.7 & $0.73 \pm 0.16$ \\
146 & $5: 39: 12.64$ & $-2: 26: 09.2$ & 5.2 & $1.91 \pm 0.45$ \\
152 & $5: 39: 16.50$ & $-2: 32: 37.2$ & 12.2 & $1.83 \pm 0.24$ \\
155 & $5: 39: 17.54$ & $-2: 33: 13.8$ & 14.0 & $2.14 \pm 0.27$ \\
158 & $5: 39: 18.90$ & $-2: 45: 23.2$ & 12.2 & $2.29 \pm 0.33$ \\
162 & $5: 39: 23.66$ & $-2: 34: 48.0$ & 9.2 & $1.47 \pm 0.25$ \\
163 & $5: 39: 24.13$ & $-2: 28: 21.9$ & 16.0 & $3.79 \pm 0.44$ \\
166 & $5: 39: 26.00$ & $-2: 44: 43.7$ & 10.7 & $2.27 \pm 0.36$ \\
168 & $5: 39: 29.49$ & $-2: 41: 02.0$ & 13.4 & $2.26 \pm 0.32$ \\
171 & $5: 39: 36.21$ & $-2: 28: 25.2$ & 6.5 & $2.01 \pm 0.51$ \\
\hline
\end{tabular}

